\title{
WEIGHTED POINCARÉ INEQUALITY AND RIGIDITY OF COMPLETE MANIFOLDS
}

\author{
BY PETER LI ${ }^{1}$ AND JiAPING WANG ${ }^{2}$
}

\begin{abstract}
We prove structure theorems for complete manifolds satisfying both the Ricci curvature lower bound and the weighted Poincaré inequality. In the process, a sharp decay estimate for the minimal positive Green's function is obtained. This estimate only depends on the weight function of the Poincaré inequality, and yields a criterion of parabolicity of connected components at infinity in terms of the weight function.
\end{abstract}

(C) 2007 Elsevier Masson SAS

RÉSUMÉ. - Nous prouvons des théorèmes de structure pour des variétés complètes telles que la courbure de Ricci soit minorée, et satisfaisant l'inégalité de Poincaré à poids. Nous obtenons une estimation optimale de la décroissance de la fonction de Green positive et minimale. Cette estimation, qui dépend seulement du poids de la fonction dans l'inégalité de Poincaré, produit un critère de parabolicité de composantes connexes à l'infini utilisant le poids de la fonction.

(c) 2007 Elsevier Masson SAS

\section{Contents}

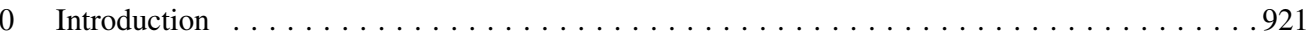

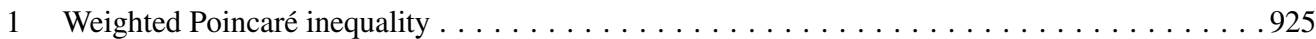

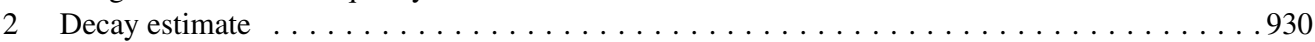

3 Geometric conditions for parabolicity and nonparabolicity . . . . . . . . . . . . . . . . 941

4 Improved Bochner formula and metric rigidity . . . . . . . . . . . . . . . . . . . . . . . . . . . 944

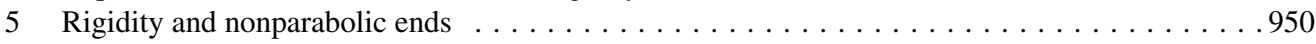

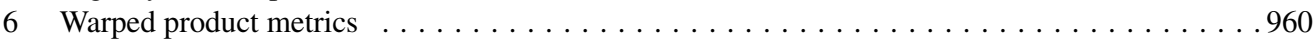

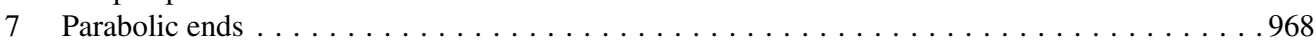

8 Nonexistence results for parabolic ends $\ldots \ldots \ldots \ldots \ldots \ldots \ldots \ldots \ldots \ldots$

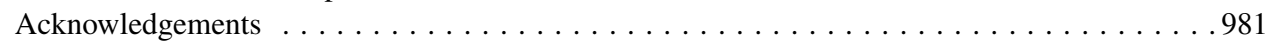

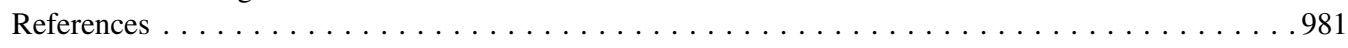

\section{Introduction}

Understanding the relations among the curvature, the topology and the function theory is a central theme in Riemannian geometry. Typically, one assumes the curvature to be bounded

${ }^{1}$ The first author was partially supported by NSF Grant DMS-0503735.

${ }^{2}$ The second author was partially supported by NSF Grant DMS-0404817. 
by a constant so that the comparison theorems become available. The main focus of the current paper, however, is to go beyond this realm to consider manifolds with Ricci curvature bounded below by a function. We will establish some structure theorems for such manifolds satisfying the following Poincaré type inequality.

DEFINITION 0.1. - Let $M^{n}$ be an $n$-dimensional complete Riemannian manifold. We say that $M$ satisfies a weighted Poincaré inequality with a nonnegative weight function $\rho(x)$, if the inequality

$$
\int_{M} \rho(x) \phi^{2}(x) d V \leqslant \int_{M}|\nabla \phi|^{2} d V
$$

is valid for all compactly supported smooth function $\phi \in C_{c}^{\infty}(M)$.

Definition 0.2. - Let $M^{n}$ be an $n$-dimensional complete Riemannian manifold. We say that $M$ has property $\left(\mathcal{P}_{\rho}\right)$ if a weighted Poincaré inequality is valid on $M$ with some nonnegative weight function $\rho$. Moreover, the $\rho$-metric, defined by

$$
d s_{\rho}^{2}=\rho d s_{M}^{2}
$$

is complete.

Let $\lambda_{1}(M)$ denote the greatest lower bound of the spectrum of the Laplacian acting on $L^{2}$ functions. Then the variational principle for $\lambda_{1}(M)$ asserts the validity of the Poincare inequality, i.e.,

$$
\lambda_{1}(M) \int_{M} \phi^{2} \leqslant \int_{M}|\nabla \phi|^{2}
$$

for all compactly supported functions $\phi \in C_{c}^{\infty}(M)$. Obviously, $M$ has property $\left(\mathcal{P}_{\rho}\right)$ with weight function $\rho=\lambda_{1}(M)$ if $\lambda_{1}(M)>0$. Hence the notion of property $\left(\mathcal{P}_{\rho}\right)$ can be viewed as a generalization of the assumption $\lambda_{1}(M)>0$.

We would like to point out that the idea of considering $d s_{\rho}^{2}$ was first used by Agmon [1] in his study of eigenfunctions for the Schrödinger operators. Indeed, we will employ some of the arguments from [1] in this paper. We also remark that the weighted Poincaré inequalities in various forms have appeared in many important issues of analysis and mathematical physics. In the interesting papers [5,6], Fefferman and Phong have considered the more general weighted Sobolev type inequalities for pseudodifferential operators.

In Section 1, we will demonstrate that a complete manifold is nonparabolic if and only if it satisfies a weighted Poincaré inequality with some weight function $\rho$. Moreover, many nonparabolic manifolds satisfy property $\left(\mathcal{P}_{\rho}\right)$ and we will provide some systematic ways to find a weight function. However, we would like to point out that the weight function is obviously not unique.

It turned out that some of the crucial estimates we developed in [11] can be generalized to give a sharper version of Agmon's estimate for the Schrödinger operators developed in [1]. We believe that these estimates are interesting in their own rights and the sharp form will find more geometric applications in our further investigation. The proof of this decay estimate will be given in Section 2.

In Section 3, we will give geometric conditions (involving $\rho$ ) for an end being nonparabolic or parabolic using the decay estimate obtained in Section 2. The conditions are parallel to what we have established for the case when $\rho=\lambda_{1}(M)$ in [11]. In Section 4, we recall a generalized Bochner formula for the gradient of a harmonic function. The equality case for this inequality

4e SÉRIE - TOME $39-2006-\mathrm{N}^{\circ} 6$ 
will be discussed. Note that the generalized Bochner formula was first used by Yau in [21] and the equality case was also used previously in $[19,11]$.

In Section 5, we give the proof of a structure theorem (Theorem 5.2) for manifolds with property $\left(\mathcal{P}_{\rho}\right)$. The Ricci curvature is assumed to satisfy the inequality

$$
\operatorname{Ric}_{M}(x) \geqslant-\frac{n-1}{n-2} \rho(x)
$$

for all $x \in M$. In this theorem, a growth assumption is needed for the weight function $\rho$ in terms of the $\rho$-distance. This allows to account for those functions $\rho$ that have different growth rate with respect to the background distance in different directions. In any case, if $\rho$ is bounded then the growth assumption is fulfilled. When the dimension of $M$ is at least 4 , the assumption on $\rho$ is rather mild. In particular, if $\rho$ is a nondecreasing function of the distance to a compact set (with respect to the background metric) then the growth assumption is automatically satisfied.

In Section 6, we will study when a warped-product situation will actually occur for specific choices of $\rho$. When $\rho$ is an increasing function, we give examples of the warped-product scenario. On the other hand, we will also prove in Theorem 6.3 that when $\liminf _{r \rightarrow \infty} \rho(r)=0$, the warped-product scenario does not exist. Let us summarize the results in Sections 5 and 6 in the following theorem. We denote by $S(R)$ the maximum value of $\sqrt{\rho}$ over the geodesic ball of radius $R$ with respect to the $\rho$-metric centered at a fixed point $p$.

THEOREM A. - Let $M^{n}$ be a complete manifold with dimension $n \geqslant 4$. Assume that $M$ satisfies property $\left(\mathcal{P}_{\rho}\right)$ for some nonzero weight function $\rho \geqslant 0$. Suppose the Ricci curvature of $M$ satisfies the lower bound

$$
\operatorname{Ric}_{M}(x) \geqslant-\frac{n-1}{n-2} \rho(x)
$$

for all $x \in M$. If $\rho$ satisfies the growth estimate

$$
\liminf _{R \rightarrow \infty} S(R) \exp \left(-\frac{n-3}{n-2} R\right)=0
$$

then either

(1) $M$ has only one nonparabolic end; or

(2) $M$ has two nonparabolic ends and is given by $M=\mathbb{R} \times N$ with the warped product metric

$$
d s_{M}^{2}=d t^{2}+\eta^{2}(t) d s_{N}^{2},
$$

for some positive function $\eta(t)$, and some compact manifold $N$. Moreover, $\rho(t)$ is a function of t alone satisfying

$$
(n-2) \eta^{\prime \prime} \eta^{-1}=\rho
$$

and

$$
\liminf _{x \rightarrow \infty} \rho(x)>0 .
$$

We will state the case $n=3$ separately since the curvature condition for counting parabolic and nonparabolic ends are the same in this case. Again, the condition on the growth rate of $\rho$ is given with respect to the $\rho$-distance. When restricted to those weight functions that are nondecreasing functions of the distance to the compact set the growth assumption is simply subexponential 
growth with respect to the background distance function. Combining with the results in Section 7 for parabolic ends, the 3 -dimension case can be stated as follows:

THEOREM B. - Let $M^{3}$ be a complete manifold of dimension 3. Assume that $M$ satisfies property $\left(\mathcal{P}_{\rho}\right)$ for some nonzero weight function $\rho \geqslant 0$. Suppose the Ricci curvature of $M$ satisfies the lower bound

$$
\operatorname{Ric}_{M}(x) \geqslant-2 \rho(x)
$$

for all $x \in M$. If $\rho$ satisfies the growth estimate

$$
\liminf _{R \rightarrow \infty} S(R) R^{-1}=0,
$$

then either

(1) $M$ has only one end;

(2) $M$ has two nonparabolic ends and is given by $M=\mathbb{R} \times N$ with the warped product metric

$$
d s_{M}^{2}=d t^{2}+\eta^{2}(t) d s_{N}^{2},
$$

for some positive function $\eta(t)$, and some compact manifold $N$. Moreover, $\rho(t)$ is a function of $t$ alone satisfying

$$
\eta^{\prime \prime} \eta^{-1}=\rho
$$

and

$$
\liminf _{x \rightarrow \infty} \rho(x)>0 ; \text { or }
$$

(3) $M$ has one parabolic end and one nonparabolic end and is given by $M=\mathbb{R} \times N$ with the warped product metric

$$
d s_{M}^{2}=d t^{2}+\eta^{2}(t) d s_{N}^{2},
$$

for some positive function $\eta(t)$, and some compact manifold $N$. Moreover, $\rho(t)$ is a function of t alone satisfying

$$
\eta^{\prime \prime} \eta^{-1}=\rho
$$

and

$$
\liminf _{x \rightarrow \infty} \rho(x)>0
$$

on the nonparabolic end.

Also, for dimension $n \geqslant 4$ we proved the following:

THEOREM C. - Let $M^{n}$ be a complete manifold of dimension $n \geqslant 4$ with property $\left(\mathcal{P}_{\rho}\right)$. Suppose the Ricci curvature of $M$ satisfies the lower bound

$$
\operatorname{Ric}_{M}(x) \geqslant-\frac{4}{n-1} \rho(x)
$$

for all $x \in M$. If $\rho$ satisfies the property that

$$
\lim _{x \rightarrow \infty} \rho(x)=0,
$$

then $M$ has only one end.

$4^{\mathrm{e}}$ SÉRIE - TOME $39-2006-\mathrm{N}^{\circ} 6$ 
Finally, in the last section, Section 8 , we prove a nonexistence result indicating that for a large class of weight functions $\rho$, namely when $\rho$ is a function of the distance and satisfying $\left(\rho^{-\frac{1}{4}}\right)^{\prime \prime}(r) \geqslant 0$ for $r$ sufficiently large, there does not exist a manifold with property $\left(\mathcal{P}_{\rho}\right)$ satisfying

$$
\operatorname{Ric}_{M}(x) \geqslant-\frac{4}{n-1} \rho(x) .
$$

We also proved a theorem restricting the behavior of the warped product.

As we have pointed out earlier, if $\lambda_{1}(M)>0$, then one may take $\rho=\lambda_{1}(M)$. This special case of Theorems A and B have been the subject of our earlier work [11,12]. The results generalized the work of Witten-Yau [20], Cai-Galloway [2], and Wang [19], on conformally compact manifolds. Also, a result in the similar spirit of Theorem $\mathrm{C}$ is available for this special case (see [12]). However, it remains open to deal with more general functions $\rho$ in Theorem C.

We would also like to point out that a similar theory was proposed in [13] for Kähler manifolds where the assumption is on the holomorphic bisectional curvature instead.

\section{Weighted Poincaré inequality}

In this section, we will show that it is not difficult to find a weight function for most manifolds. The following proposition gives a convenient way to construct a weight function. The argument uses a modified version of the Barta inequality for the first eigenvalue. Using this method, we will show that a manifold is nonparabolic if and only if there exists a nontrivial weight function. We will also give examples of using other methods to obtain a weight function for various manifolds.

PROPOSITION 1.1. - Let $M$ be a complete Riemannian manifold. If there exists a nonnegative function $h$ defined on $M$, that is not identically 0 , satisfying

$$
\Delta h(x) \leqslant-\rho(x) h(x),
$$

then the weighted Poincaré inequality

$$
\int_{M} \rho(x) \phi^{2}(x) \leqslant \int_{M}|\nabla \phi|^{2}(x)
$$

must be valid for all compactly supported smooth function $\phi \in C_{c}^{\infty}(M)$.

Proof. - Let $D \subset M$ be a smooth compact subdomain of $M$. Let us denote $\lambda_{1}(\rho, D)$ to be the first Dirichlet eigenvalue on $D$ for the operator

$$
\Delta+\rho(x)
$$

Let $u$ be the first eigenfunction satisfying

$$
\Delta u(x)+\rho(x) u(x)=-\lambda_{1}(\rho, D) u(x) \quad \text { on } D
$$

and

$$
u(x)=0 \quad \text { on } \partial D .
$$


We may assume that $u \geqslant 0$ on $D$, and the regularity of $u$ asserts that $u>0$ in the interior of $D$. Integration by parts yields

$$
\int_{D} u \Delta h-\int_{D} h \Delta u=\int_{\partial D} u \frac{\partial h}{\partial \nu}-\int_{\partial D} h \frac{\partial u}{\partial \nu} \geqslant 0,
$$

where $\nu$ is the outward unit normal of $\partial D$. On the other hand, the assumption on $h$ implies that

$$
u \Delta h-h \Delta u \leqslant \lambda_{1}(\rho, D) u h .
$$

Since both $u>0$ and $h$ are not identically 0 , this combining with (1.1) implies that $\lambda_{1}(\rho, D) \geqslant 0$. In particular, the variational characterization of $\lambda_{1}(\rho, D)$ implies that

$$
\begin{aligned}
0 & \leqslant \lambda_{1}(\rho, D) \int_{D} \phi^{2}(x) \\
& \leqslant \int_{D}|\nabla \phi|^{2}-\int_{D} \rho(x) \phi^{2}(x)
\end{aligned}
$$

for all $\phi$ with support in $D$. Since $D$ is arbitrary, this implies the weighted Poincaré inequality.

Let us now quickly recall the definition of parabolicity. Full detail on the discussion of parabolicity can be found in [8].

DEFINITION 1.2. - A complete manifold $M$ is said to be nonparabolic if there exists a symmetric positive Green's function $G(x, y)$ for the Laplacian acting on $L^{2}$ functions. Otherwise, we say that $M$ is parabolic.

Similarly, the notion of parabolicity is also valid when localized at an end of a manifold. We recall (see [8]) that an end is simply an unbounded component of $M \backslash \Omega$, where $\Omega$ is a compact smooth domain of $M$.

DEFINITION 1.3. - Let $E$ be an end of a complete manifold $M$. We say that $E$ is nonparabolic if there exists a symmetric, positive, Green's function $G(x, y)$ for the Laplacian acting on $L^{2}$ functions with Neumann boundary condition on $\partial E$. Otherwise, we say that $E$ is a parabolic end.

Note that (see [8]) a complete manifold is nonparabolic if and only if $M$ has at least one nonparabolic end. More importantly, it is possible for a nonparabolic manifold to have many parabolic ends. It is also known that [8] a manifold is parabolic if we consider the sequence of harmonic functions $\left\{f_{i}\right\}$ defined on $B\left(R_{i}\right) \backslash B\left(R_{0}\right)$ satisfying

$$
\Delta f_{i}=0 \quad \text { on } B\left(R_{i}\right) \backslash B\left(R_{0}\right)
$$

with boundary conditions

$$
f_{i}= \begin{cases}1 & \text { on } \partial B\left(R_{0}\right), \\ 0 & \text { on } \partial B\left(R_{i}\right),\end{cases}
$$

then they converge to the constant function $f=1$ defined on $M \backslash B\left(R_{0}\right)$ as $R_{i} \rightarrow \infty$ for any fixed $R_{0}$.

$4^{\text {e }}$ SÉRIE - TOME $39-2006-\mathrm{N}^{\circ} 6$ 
COROLLARY 1.4. - Let $M$ be a complete nonparabolic manifold and $G(p, x)$ be the minimal positive Green's function defined on $M$ with a pole at the point $p \in M$. Then $M$ satisfies the weighted Poincare inequality with the weight function $\rho$ given by

$$
\rho(x)=\frac{|\nabla G(p, x)|^{2}}{4 G^{2}(p, x)} .
$$

Conversely, if a nonzero weight function $\rho \geqslant 0$ exists, then $M$ must be nonparabolic.

Proof. - Let us first assume that $M$ is nonparabolic and hence a positive symmetric Green's function, $G(x, y)$, for the Laplacian exists. Let $p \in M$ be a fixed point and

$$
g_{a}(x)=\min \{a, G(p, x)\} .
$$

Then $g_{a}$ is a superharmonic function defined on $M$. A direct computation yields that

$$
\Delta g_{a}^{\frac{1}{2}} \leqslant-\frac{\left|\nabla g_{a}\right|^{2}}{4 g_{a}^{2}} g_{a}^{\frac{1}{2}}
$$

on $M$. Hence Proposition 1.1 asserts that the weighted Poincaré inequality is valid with

$$
\rho=\frac{\left|\nabla g_{a}\right|^{2}}{4 g_{a}^{2}} .
$$

Letting $a \rightarrow \infty$, we conclude that we can take

$$
\rho(x)=\frac{|\nabla G(p, x)|^{2}}{4 G^{2}(p, x)} .
$$

This proves the first part of the corollary.

Conversely, let us assume that the weighted Poincaré inequality is valid for a nonzero weight function $\rho \geqslant 0$. Assuming on the contrary that $M$ is parabolic, we will find a contradiction. Indeed, if $M$ is parabolic then let us consider the sequence of compactly supported functions

$$
\phi_{i}= \begin{cases}1 & \text { on } B\left(R_{0}\right), \\ f_{i} & \text { on } B\left(R_{i}\right) \backslash B\left(R_{0}\right), \\ 0 & \text { on } M \backslash B\left(R_{i}\right)\end{cases}
$$

where $f_{i}$ is given by the sequence of harmonic functions obtained from (1.2) and (1.3). Setting $\phi=\phi_{i}$ in the weighted Poincaré inequality, we have

$$
\begin{aligned}
\int_{M} \rho \phi_{i}^{2} & \leqslant \int_{M}\left|\nabla \phi_{i}\right|^{2} \\
& =\int_{B\left(R_{i}\right) \backslash B\left(R_{0}\right)}\left|\nabla f_{i}\right|^{2} \\
& =\int_{\partial B\left(R_{i}\right)} f_{i} \frac{\partial f_{i}}{\partial \nu}-\int_{\partial B\left(R_{0}\right)} f_{i} \frac{\partial f_{i}}{\partial \nu}
\end{aligned}
$$

ANNALES SCIENTIFIQUES DE L'ÉCOLE NORMALE SUPÉRIEURE 


$$
=-\int_{\partial B\left(R_{0}\right)} \frac{\partial f_{i}}{\partial \nu} .
$$

However, since $f_{i} \rightarrow 1$ on $M \backslash B\left(R_{0}\right)$, we conclude that

$$
\int_{M} \rho \leqslant 0,
$$

violating the assumption that $\rho \geqslant 0$ is nonzero. This proves the second part of the corollary.

While the existence of a weighted Poincare inequality is equivalent to nonparabolicity, the condition that $M$ has property $\left(\mathcal{P}_{\rho}\right)$ is not as clear cut. The following lemma gives a sufficient condition for $\left(\mathcal{P}_{\rho}\right)$.

LEMMA 1.5. - Let $M$ be a nonparabolic complete manifold. Suppose $G(x, y)$ is the minimal, symmetric, positive, Green's function for the Laplacian acting on $L^{2}$ functions. For a fixed point $p \in M$, if $G(p, x) \rightarrow 0$ as $x \rightarrow \infty$, then $M$ has property $\left(\mathcal{P}_{\rho}\right)$ with

$$
\rho=\frac{|\nabla G(p, x)|^{2}}{4 G^{2}(p, x)} .
$$

Proof. - In view of Corollary 1.4, it remains to show that the $\rho$-metric is complete. Indeed, let $\gamma(s)$ be a curve parametrized by arclength, $0 \leqslant s \leqslant T$. The length of $\gamma$ with respect to the $\rho$-metric is given by

$$
\int_{\gamma} \sqrt{\rho} d s=\frac{1}{2} \int_{\gamma}|\nabla \log G| d s .
$$

However, since

$$
\begin{aligned}
\log G(p, \gamma(0))-\log G(p, \gamma(T)) & =-\int_{0}^{T} \frac{\partial}{\partial s}(\log G(p, \gamma(s))) d s \\
& \leqslant \int_{\gamma}|\nabla \log G| d s,
\end{aligned}
$$

we conclude that the $\rho$-length of $\gamma$ is infinity if $\gamma(T) \rightarrow \infty$. This proves the completeness of the $\rho$-metric.

We should point out that it is not necessarily true that if $G(p, x)$ does not tend to 0 at infinity then $M$ does not have property $\left(\mathcal{P}_{\rho}\right)$ since there might be another weight function that gives a complete metric.

Example 1.6. - When $M=\mathbb{R}^{n}$ for $n \geqslant 3$, the Green's function is given by

$$
G(0, x)=C_{n} r^{2-n}(x)
$$

for some constant $C_{n}>0$ depending only on $n$. In this case, we compute that

$$
\frac{|\nabla G(0, x)|^{2}}{4 G^{2}(0, x)}=\frac{(n-2)^{2}}{4} r^{-2}(x) .
$$

$4^{\text {e }}$ SÉRIE - TOME $39-2006-\mathrm{N}^{\circ} 6$ 
Hence from the above discussion, we conclude that the weighted Poincaré inequality

$$
\frac{(n-2)^{2}}{4} \int_{\mathbb{R}^{n}} r^{-2} \phi^{2} \leqslant \int_{\mathbb{R}^{n}}|\nabla \phi|^{2},
$$

which is the well-known Hardy's inequality, must be valid for all compactly supported smooth function $\phi \in C_{c}^{\infty}\left(\mathbb{R}^{n}\right)$, and $\mathbb{R}^{n}$ has property $\left(\mathcal{P}_{\rho}\right)$ with

$$
\rho(x)=\frac{(n-2)^{2}}{4} r^{-2}(x)
$$

Example 1.7. - Let $M^{n}$ be a minimal submanifold of dimension $n \geqslant 3$ in $\mathbb{R}^{N}$. If we denote $\bar{r}$ to be the extrinsic distance function of $\mathbb{R}^{N}$ to a fixed point $p \in M$, then it is known that it satisfies the equation

$$
\Delta \bar{r} \geqslant(n-1) \bar{r}^{-1},
$$

where $\Delta$ is the Laplacian on $M$ with respect to the induced metric from $\mathbb{R}^{N}$. For any $\phi \in$ $C_{c}^{\infty}(M)$, we consider the integral

$$
\begin{aligned}
\int_{M} \bar{r}^{-1} \phi^{2} \Delta \bar{r} & =-2 \int_{M} \bar{r}^{-1} \phi\langle\nabla \phi, \nabla \bar{r}\rangle+\int_{M} \bar{r}^{-2} \phi^{2}|\nabla \bar{r}|^{2} \\
& \leqslant 2 \int_{M} \bar{r}^{-1} \phi|\nabla \phi|+\int_{M} \bar{r}^{-2} \phi^{2} .
\end{aligned}
$$

Combining with (1.4), this implies that

$$
\begin{aligned}
\frac{(n-2)}{2} \int_{M} \bar{r}^{-2} \phi^{2} & \leqslant \int_{M} \bar{r}^{-1} \phi|\nabla \phi| \\
& \leqslant\left(\int_{M} \bar{r}^{-2} \phi^{2}\right)^{\frac{1}{2}}\left(\int_{M}|\nabla \phi|^{2}\right)^{\frac{1}{2}},
\end{aligned}
$$

hence the weighted Poincaré inequality is valid on $M$ with

$$
\rho=\frac{(n-2)^{2}}{4} \bar{r}^{-2} .
$$

If we further assume that $M$ is properly immersed, then Lemma 1.5 implies that $M$ has property $\left(\mathcal{P}_{\rho}\right)$.

Example 1.8. - Let $M$ be a simply connected, complete, Cartan-Hadamard manifold with sectional curvature bounded from above by

$$
K_{M} \leqslant-1 .
$$

In this case, the Hessian comparison theorem asserts that

$$
\Delta r \geqslant(n-1) \operatorname{coth} r
$$


where $r$ is the geodesic distance function from a fixed point $p \in M$. Suppose $\phi \in C_{c}^{\infty}(M)$ is a compactly supported function. Then (1.5) and integration by parts yield

$$
\begin{aligned}
(n-1) \int_{M} \phi^{2} \operatorname{coth} r & \leqslant \int_{M} \phi^{2} \Delta r \\
& =-2 \int_{M} \phi\langle\nabla \phi, \nabla r\rangle \\
& \leqslant 2 \int_{M} \phi|\nabla \phi| \\
& \leqslant \frac{n-1}{2} \int_{M} \phi^{2}+\frac{2}{n-1} \int_{M}|\nabla \phi|^{2} .
\end{aligned}
$$

This can be rewritten as

$$
\frac{(n-1)^{2}}{4} \int_{M} \phi^{2}+\frac{(n-1)^{2}}{2} \int_{M}(\operatorname{coth} r-1) \phi^{2} \leqslant \int_{M}|\nabla \phi|^{2} .
$$

Hence in this case, the weight function $\rho$ can be taken to be

$$
\begin{aligned}
\rho & =\frac{(n-1)^{2}}{4}+\frac{(n-1)^{2}}{2}(\operatorname{coth} r-1) \\
& \geqslant \frac{(n-1)^{2}}{4} .
\end{aligned}
$$

Since it is bounded from below by a positive constant, the $\rho$-metric must be complete by the completeness assumption on $d s_{M}^{2}$, and $M$ has property $\left(\mathcal{P}_{\rho}\right)$.

\section{Decay estimate}

In this section, we will consider a more general situation. Let $V$ be a given potential function defined on $M$, and

$$
\Delta-V(x)
$$

be the Schrödinger operator on $M$. We assume that there exists a positive function $\rho$ defined on $M$, such that the weighted Poincaré type inequality

$$
\int_{M} \rho(x) \phi^{2}(x) d x \leqslant \int_{M}|\nabla \phi|^{2}(x) d x+\int_{M} V(x) \phi^{2}(x) d x
$$

is valid for any compactly supported function $\phi \in C_{c}^{\infty}(M)$. Let us define the $\rho$-metric given by

$$
d s_{\rho}^{2}=\rho d s^{2} .
$$

Using this metric, we consider the $\rho$-distance function defined to be,

$$
r_{\rho}(x, y)=\inf _{\gamma} \ell_{\rho}(\gamma)
$$

$4^{\mathrm{e}}$ SÉRIE - TOME $39-2006-\mathrm{N}^{\circ} 6$ 
the infimum of the lengths of all smooth curves joining $x$ and $y$ with respect to $d s_{\rho}^{2}$. For a fixed point $p \in M$, we denote $r_{\rho}(x)=r_{\rho}(p, x)$ to be the $\rho$-distance to $p$. One checks readily that $\left|\nabla r_{\rho}\right|^{2}(x)=\rho(x)$. As in the case when $V=0$, we say that the manifold has property $\left(\mathcal{P}_{\rho, V}\right)$ if the $\rho$-metric is complete, and this will be the standing assumption on that $M$.

Throughout this article, we denote

$$
B_{\rho}(p, R)=\left\{x \in M \mid r_{\rho}(p, x)<R\right\}
$$

to be the set of points in $M$ that has $\rho$-distance less than $R$ from point $p$. We also denote

$$
B(p, R)=\{x \in M \mid r(p, x)<R\}
$$

to be the set of points in $M$ that has distance less than $R$ from point $p$ with respect to the background metric $d s_{M}^{2}$. When $p \in M$ is a fixed point, we will suppress the dependency of $p$ and write $B_{\rho}(R)=B_{\rho}(p, R)$ and $B(R)=B(p, R)$. If $E$ is an end of $M$, we denote $E_{\rho}(R)=B_{\rho}(R) \cap E$.

THEOREM 2.1. - Let $M$ be a complete Riemannian manifold with property $\left(\mathcal{P}_{\rho, V}\right)$. Suppose $E$ is an end of $M$ such that there exists a nonnegative function $\rho(x)$ defined on $E$ with the property that

$$
\int_{E} \rho(x) \phi^{2}(x) d x \leqslant \int_{E}|\nabla \phi|^{2}(x) d x+\int_{E} V(x) \phi^{2}(x) d x
$$

for any compactly supported function $\phi \in C_{c}^{\infty}(E)$. Let $f$ be a nonnegative function defined on $E$ satisfying the differential inequality

$$
(\Delta-V(x)) f(x) \geqslant 0 .
$$

If $f$ satisfies the growth condition

$$
\int_{E_{\rho}(R)} \rho f^{2} \exp \left(-2 r_{\rho}\right)=o(R)
$$

as $R \rightarrow \infty$, then it must satisfy the decay estimate

$$
\int_{E_{\rho}(R+1) \backslash E_{\rho}(R)} \rho f^{2} \leqslant C \exp (-2 R)
$$

for some constant $C>0$ depending on $f$ and $\rho$.

Proof. - We will first prove that for any $0<\delta<1$, there exists a constant $0<C<\infty$ such that,

$$
\int_{E} \rho \exp \left(2 \delta r_{\rho}\right) f^{2} \leqslant C .
$$

Indeed, let $\phi\left(r_{\rho}(x)\right)$ be a nonnegative cut-off function with support in $E$ with $r_{\rho}(x)$ being the $\rho$-distance to the fixed point $p$. Then for any function $h\left(r_{\rho}(x)\right)$ integration by parts yields 


$$
\begin{aligned}
\int_{E} \mid \nabla & \left.(\phi f \exp (h))\right|^{2} \\
= & \int_{E}|\nabla(\phi \exp (h))|^{2} f^{2}+\int_{E}(\phi \exp (h))^{2}|\nabla f|^{2} \\
& +2 \int_{E} \phi f \exp (h)\langle\nabla(\phi \exp (h)), \nabla f\rangle \\
= & \int_{E}|\nabla(\phi \exp (h))|^{2} f^{2}+\int_{E} \phi^{2} \exp (2 h)|\nabla f|^{2} \\
& +\frac{1}{2} \int_{E}\left\langle\nabla\left(\phi^{2} \exp (2 h)\right), \nabla\left(f^{2}\right)\right\rangle \\
= & \int_{E}|\nabla(\phi \exp (h))|^{2} f^{2}+\int_{E} \phi^{2} \exp (2 h)|\nabla f|^{2}-\frac{1}{2} \int_{E} \phi^{2} \exp (2 h) \Delta\left(f^{2}\right) \\
= & \int_{E}|\nabla(\phi \exp (h))|^{2} f^{2}-\int_{E} \phi^{2} \exp (2 h) f \Delta f \\
\leqslant & \int_{E}|\nabla(\phi \exp (h))|^{2} f^{2}-\int_{E} V \phi^{2} \exp (2 h) f^{2} \\
= & \int_{E}|\nabla \phi|^{2} f^{2} \exp (2 h)+2 \int_{E} \phi \exp (2 h)\langle\nabla \phi, \nabla h\rangle f^{2} \\
& +\int_{E} \phi^{2}|\nabla h|^{2} f^{2} \exp (2 h)-\int_{E} V \phi^{2} f^{2} \exp (2 h) .
\end{aligned}
$$

On the other hand, using the assumption (2.1), we have

$$
\int_{E} \rho \phi^{2} f^{2} \exp (2 h) \leqslant \int_{E}|\nabla(\phi f \exp (h))|^{2}+\int_{E} V \phi^{2} f^{2} \exp (2 h),
$$

hence (2.2) becomes

$$
\begin{aligned}
\int_{E} \rho \phi^{2} f^{2} \exp (2 h) \leqslant & \int_{E}|\nabla \phi|^{2} f^{2} \exp (2 h)+2 \int_{E} \phi \exp (2 h)\langle\nabla \phi, \nabla h\rangle f^{2} \\
& +\int_{E} \phi^{2}|\nabla h|^{2} f^{2} \exp (2 h) .
\end{aligned}
$$

Let us now choose

$$
\phi\left(r_{\rho}(x)\right)= \begin{cases}r_{\rho}(x)-R_{0} & \text { on } E_{\rho}\left(R_{0}+1\right) \backslash E_{\rho}\left(R_{0}\right), \\ 1 & \text { on } E_{\rho}(R) \backslash E_{\rho}\left(R_{0}+1\right), \\ R^{-1}\left(2 R-r_{\rho}(x)\right) & \text { on } E_{\rho}(2 R) \backslash E_{\rho}(R), \\ 0 & \text { on } E \backslash E_{\rho}(2 R),\end{cases}
$$


and hence

$$
|\nabla \phi|^{2}(x)= \begin{cases}\rho(x) & \text { on } E_{\rho}\left(R_{0}+1\right) \backslash E_{\rho}\left(R_{0}\right), \\ R^{-2} \rho(x) & \text { on } E_{\rho}(2 R) \backslash E_{\rho}(R), \\ 0 & \text { on }\left(E_{\rho}(R) \backslash E_{\rho}\left(R_{0}+1\right)\right) \cup\left(E \backslash E_{\rho}(2 R)\right) .\end{cases}
$$

We also choose

$$
h\left(r_{\rho}(x)\right)= \begin{cases}\delta r_{\rho}(x) & \text { for } r_{\rho} \leqslant \frac{K}{(1+\delta)}, \\ K-r_{\rho}(x) & \text { for } r_{\rho} \geqslant \frac{K}{(1+\delta)},\end{cases}
$$

for some fixed $K>\left(R_{0}+1\right)(1+\delta)$. When $R \geqslant \frac{K}{(1+\delta)}$, we see that

$$
|\nabla h|^{2}(x)= \begin{cases}\delta^{2} \rho(x) & \text { for } r_{\rho} \leqslant \frac{K}{(1+\delta)} \\ \rho(x) & \text { for } r_{\rho} \geqslant \frac{K}{(1+\delta)}\end{cases}
$$

and

$$
\langle\nabla \phi, \nabla h\rangle(x)= \begin{cases}\delta \rho(x) & \text { on } E_{\rho}\left(R_{0}+1\right) \backslash E_{\rho}\left(R_{0}\right), \\ R^{-1} \rho(x) & \text { on } E_{\rho}(2 R) \backslash E_{\rho}(R), \\ 0 & \text { otherwise. }\end{cases}
$$

Substituting into (2.3), we obtain

$$
\begin{aligned}
\int_{E} \rho \phi^{2} f^{2} \exp (2 h) \leqslant & \int_{E_{\rho}\left(R_{0}+1\right) \backslash E_{\rho}\left(R_{0}\right)} \rho f^{2} \exp (2 h)+R^{-2} \int_{E_{\rho}(2 R) \backslash E_{\rho}(R)} \rho f^{2} \exp (2 h) \\
& +2 \delta \int_{E_{\rho}\left(R_{0}+1\right) \backslash E_{\rho}\left(R_{0}\right)} \rho f^{2} \exp (2 h) \\
& +2 R^{-1} \int_{E_{\rho}(2 R) \backslash E_{\rho}(R)} \rho f^{2} \exp (2 h) \\
& +\delta^{2} \int_{E_{\rho}\left(K(1+\delta)^{-1}\right) \backslash E_{\rho}\left(R_{0}\right)} \rho \phi^{2} f^{2} \exp (2 h) \\
& +\int_{E_{\rho}(2 R) \backslash E_{\rho}\left(K(1+\delta)^{-1}\right)} \rho \phi^{2} f^{2} \exp (2 h) .
\end{aligned}
$$

This can be rewritten as

$$
\begin{aligned}
\int_{E_{\rho}\left(K(1+\delta)^{-1} \backslash \backslash E_{\rho}\left(R_{0}+1\right)\right.} \rho f^{2} \exp (2 h) \leqslant & \int_{E_{\rho}\left(K(1+\delta)^{-1}\right)} \rho \phi^{2} f^{2} \exp (2 h) \\
\leqslant & \int_{E_{\rho}\left(R_{0}+1\right) \backslash E_{\rho}\left(R_{0}\right)} \rho f^{2} \exp (2 h) \\
& +R^{-2} \int_{E_{\rho}(2 R) \backslash E_{\rho}(R)} \rho f^{2} \exp (2 h)
\end{aligned}
$$

ANNALES SCIENTIFIQUES DE L'ÉCOLE NORMALE SUPÉRIEURE 


$$
\begin{aligned}
& +2 \delta \int_{E_{\rho}\left(R_{0}+1\right) \backslash E_{\rho}\left(R_{0}\right)} \rho f^{2} \exp (2 h) \\
& +2 R^{-1} \int_{E_{\rho}(2 R) \backslash E_{\rho}(R)} \rho f^{2} \exp (2 h) \\
& +\delta^{2} \int_{E_{\rho}\left(K(1+\delta)^{-1}\right) \backslash E_{\rho}\left(R_{0}\right)} \rho f^{2} \exp (2 h),
\end{aligned}
$$

hence

$$
\begin{aligned}
& \left(1-\delta^{2}\right) \underset{E_{\rho}\left(K(1+\delta)^{-1}\right) \backslash E_{\rho}\left(R_{0}+1\right)}{\rho} \rho f^{2} \exp (2 h) \\
& \leqslant\left(\delta^{2}+2 \delta+1\right) \int_{E_{\rho}\left(R_{0}+1\right) \backslash E_{\rho}\left(R_{0}\right)} \rho f^{2} \exp (2 h) \\
& +R^{-2} \int_{E_{\rho}(2 R) \backslash E_{\rho}(R)} \rho f^{2} \exp (2 h)+2 R^{-1} \int_{E_{\rho}(2 R) \backslash E_{\rho}(R)} \rho f^{2} \exp (2 h) .
\end{aligned}
$$

The definition of $h$ and the assumption on the growth condition on $f$ imply that the last two terms on the right-hand side tend to 0 as $R \rightarrow \infty$. Hence we obtain the estimate

$$
\begin{aligned}
& \left(1-\delta^{2}\right) \int_{E_{\rho}\left(K(1+\delta)^{-1}\right) \backslash E_{\rho}\left(R_{0}+1\right)} \rho f^{2} \exp \left(2 \delta r_{\rho}\right) \\
& \leqslant\left(\delta^{2}+2 \delta+1\right) \int_{E_{\rho}\left(R_{0}+1\right) \backslash E_{\rho}\left(R_{0}\right)} \rho f^{2} \exp \left(2 \delta r_{\rho}\right) .
\end{aligned}
$$

Since the right-hand side is independent of $K$, by letting $K \rightarrow \infty$ we conclude that

$$
\int_{E \backslash E_{\rho}\left(R_{0}+1\right)} \rho f^{2} \exp \left(2 \delta r_{\rho}\right) \leqslant C,
$$

for some constant $0<C<\infty$.

Our next step is to improve this estimate by setting $h=r_{\rho}$ in the preceding argument. Note that with this choice of $h,(2.3)$ asserts that

$$
-2 \int_{E} \phi \exp \left(2 r_{\rho}\right)\left\langle\nabla \phi, \nabla r_{\rho}\right\rangle f^{2} \leqslant \int_{E}|\nabla \phi|^{2} f^{2} \exp \left(2 r_{\rho}\right) .
$$

For $R_{0}<R_{1}<R$, let us choose $\phi$ to be

$$
\phi(x)= \begin{cases}\frac{r_{\rho}(x)-R_{0}}{R_{1}-R_{0}} & \text { on } E_{\rho}\left(R_{1}\right) \backslash E_{\rho}\left(R_{0}\right), \\ \frac{R-r_{\rho}(x)}{R-R_{1}} & \text { on } E_{\rho}(R) \backslash E_{\rho}\left(R_{1}\right) .\end{cases}
$$


We conclude that

$$
\begin{aligned}
& \frac{2}{R-R_{1}} \int_{E_{\rho}(R) \backslash E_{\rho}\left(R_{1}\right)}\left(\frac{R-r_{\rho}(x)}{R-R_{1}}\right) \rho f^{2} \exp \left(2 r_{\rho}\right) \\
& \leqslant \frac{1}{\left(R_{1}-R_{0}\right)^{2}} \int_{E_{\rho}\left(R_{1}\right) \backslash E_{\rho}\left(R_{0}\right)} \rho f^{2} \exp \left(2 r_{\rho}\right) \\
& \quad+\frac{1}{\left(R-R_{1}\right)^{2}} \int_{E_{\rho}(R) \backslash E_{\rho}\left(R_{1}\right)} \rho f^{2} \exp \left(2 r_{\rho}\right) \\
& \quad+\frac{2}{\left(R_{1}-R_{0}\right)^{2}} \int_{E_{\rho}\left(R_{1}\right) \backslash E_{\rho}\left(R_{0}\right)}\left(r_{\rho}-R_{0}\right) \rho f^{2} \exp \left(2 r_{\rho}\right) .
\end{aligned}
$$

On the other hand, for any $0<t<R-R_{1}$, since

$$
\begin{aligned}
& \frac{2 t}{\left(R-R_{1}\right)^{2}} \int_{E_{\rho}(R-t) \backslash E_{\rho}\left(R_{1}\right)} \rho f^{2} \exp \left(2 r_{\rho}\right) \\
& \leqslant \frac{2}{\left(R-R_{1}\right)^{2}} \int_{E_{\rho}(R) \backslash E_{\rho}\left(R_{1}\right)}\left(R-r_{\rho}(x)\right) \rho f^{2} \exp \left(2 r_{\rho}\right),
\end{aligned}
$$

we deduce that

$$
\begin{aligned}
& \frac{2 t}{\left(R-R_{1}\right)^{2}} \int_{E_{\rho}(R-t) \backslash E_{\rho}\left(R_{1}\right)} \rho f^{2} \exp \left(2 r_{\rho}\right) \\
& \leqslant\left(\frac{2}{R_{1}-R_{0}}+\frac{1}{\left(R_{1}-R_{0}\right)^{2}}\right) \int_{E_{\rho}\left(R_{1}\right) \backslash E_{\rho}\left(R_{0}\right)} \rho f^{2} \exp \left(2 r_{\rho}\right) \\
& \quad+\frac{1}{\left(R-R_{1}\right)^{2}} \int_{E_{\rho}(R) \backslash E_{\rho}\left(R_{1}\right)} \rho f^{2} \exp \left(2 r_{\rho}\right) .
\end{aligned}
$$

Observe that by taking $R_{1}=R_{0}+1, t=1$, and setting

$$
g(R)=\int_{E_{\rho}(R) \backslash E_{\rho}\left(R_{0}+1\right)} \rho f^{2} \exp \left(2 r_{\rho}\right),
$$

the inequality (2.5) can be written as

$$
g(R-1) \leqslant C_{1} R^{2}+\frac{1}{2} g(R),
$$

where

$$
C_{1}=\frac{3}{2} \int_{E_{\rho}\left(R_{0}+1\right) \backslash E_{\rho}\left(R_{0}\right)} \rho f^{2} \exp \left(2 r_{\rho}\right)
$$

is independent of $R$. Iterating this inequality, we obtain that for any positive integer $k$ and $R \geqslant 1$ 


$$
\begin{aligned}
g(R) & \leqslant C_{1} \sum_{i=1}^{k} \frac{(R+i)^{2}}{2^{i-1}}+2^{-k} g(R+k) \\
& \leqslant C_{1} R^{2} \sum_{i=1}^{\infty} \frac{(1+i)^{2}}{2^{i-1}}+2^{-k} g(R+k) \\
& \leqslant C_{2} R^{2}+2^{-k} g(R+k)
\end{aligned}
$$

for some constant $C_{2}$. However, our previous estimate (2.4) asserts that

$$
\int_{E} \rho f^{2} \exp \left(2 \delta r_{\rho}\right) \leqslant C
$$

for any $\delta<1$. This implies that

$$
\begin{aligned}
g(R+k) & =\int_{E_{\rho}(R+k) \backslash E_{\rho}\left(R_{0}+1\right)} \rho f^{2} \exp \left(2 r_{\rho}\right) \\
& \leqslant \exp (2(R+k)(1-\delta)) \int_{E_{\rho}(R+k) \backslash E_{\rho}\left(R_{0}+1\right)} \rho f^{2} \exp \left(2 \delta r_{\rho}\right) \\
& \leqslant C \exp (2(R+k)(1-\delta)) .
\end{aligned}
$$

Hence,

$$
2^{-k} g(R+k) \rightarrow 0
$$

as $k \rightarrow \infty$ by choosing $2(1-\delta)<\ln 2$. This proves the estimate

$$
g(R) \leqslant C_{2} R^{2}
$$

By adjusting the constant, we have

$$
\int_{E_{\rho}(R)} \rho f^{2} \exp \left(2 r_{\rho}\right) \leqslant C_{3} R^{2}
$$

for all $R \geqslant R_{0}$.

Using inequality (2.5) again and by choosing $R_{1}=R_{0}+1$ and $t=\frac{R}{2}$ this time, we conclude that

$$
R \int_{E_{\rho}\left(\frac{R}{2}\right) \backslash E_{\rho}\left(R_{0}+1\right)} \rho f^{2} \exp \left(2 r_{\rho}\right) \leqslant C_{4} R^{2}+\int_{E_{\rho}(R) \backslash E_{\rho}\left(R_{0}+1\right)} \rho f^{2} \exp \left(2 r_{\rho}\right) .
$$

However, applying the estimate (2.6) to the second term on the right-hand side, we have

$$
\int_{E_{\rho}\left(\frac{R}{2}\right) \backslash E_{\rho}\left(R_{0}+1\right)} \rho f^{2} \exp \left(2 r_{\rho}\right) \leqslant C_{5} R .
$$

Therefore, for $R \geqslant R_{0}$,

$$
\int_{E_{\rho}(R)} \rho f^{2} \exp \left(2 r_{\rho}\right) \leqslant C R
$$

$4^{\mathrm{e}}$ SÉRIE - TOME $39-2006-\mathrm{N}^{\circ} 6$ 
We are now ready to prove the theorem by using (2.7). Setting $t=2$ and $R_{1}=R-4$ in (2.5), we obtain

$$
\begin{aligned}
& \int_{E_{\rho}(R-2) \backslash E_{\rho}(R-4)} \rho f^{2} \exp \left(2 r_{\rho}\right) \\
& \leqslant\left(\frac{8}{R-R_{0}-4}+\frac{4}{\left(R-R_{0}-4\right)^{2}}\right) \int_{E_{\rho}(R-4) \backslash E_{\rho}\left(R_{0}\right)} \rho f^{2} \exp \left(2 r_{\rho}\right) \\
& \quad+\frac{1}{4} \int_{E_{\rho}(R) \backslash E_{\rho}(R-4)} \rho f^{2} \exp \left(2 r_{\rho}\right) .
\end{aligned}
$$

According to (2.7), the first term of the right-hand side is bounded by a constant. Hence, the above inequality can be rewritten as

$$
\int_{E_{\rho}(R-2) \backslash E_{\rho}(R-4)} \rho f^{2} \exp \left(2 r_{\rho}\right) \leqslant C+\frac{1}{3} \int_{E_{\rho}(R) \backslash E_{\rho}(R-2)} \rho f^{2} \exp \left(2 r_{\rho}\right) .
$$

Iterating this inequality $k$ times, we arrive at

$$
\begin{aligned}
& \int_{E_{\rho}(R+2) \backslash E_{\rho}(R)} \rho f^{2} \exp \left(2 r_{\rho}\right) \\
& \leqslant C \sum_{i=0}^{k-1} 3^{-i}+3^{-k} \int_{E_{\rho}(R+2(k+1)) \backslash E_{\rho}(R+2 k)} \rho f^{2} \exp \left(2 r_{\rho}\right) .
\end{aligned}
$$

However, using (2.7) again, we conclude that the second term is bounded by

$$
3^{-k} \int_{E_{\rho}(R+2(k+1)) \backslash E_{\rho}(R+2 k)} \rho f^{2} \exp \left(2 r_{\rho}\right) \leqslant C 3^{-k}(R+2(k+1))
$$

which tends to 0 as $k \rightarrow \infty$. Hence

$$
\int_{E_{\rho}(R+2) \backslash E_{\rho}(R)} \rho f^{2} \exp \left(2 r_{\rho}\right) \leqslant C
$$

for some constant $C>0$ independent of $R$. The theorem now follows from (2.8).

We now draw some corollaries.

COROllary 2.2. - Let $M$ be a complete Riemannian manifold. Suppose $E$ is an end of $M$ such that $\lambda_{1}(E)>0$, i.e.,

$$
\lambda_{1}(E) \int_{E} \phi^{2}(x) d x \leqslant \int_{E}|\nabla \phi|^{2}(x) d x
$$

for any compactly supported function $\phi \in C_{c}^{\infty}(E)$. Let $f$ be a nonnegative function defined on E satisfying the differential inequality

$$
(\Delta+\mu) f(x) \geqslant 0
$$


for some constant $\mu$ with the property that $\lambda_{1}(E)-\mu>0$. If $a=\sqrt{\lambda_{1}(E)-\mu}$ and $f$ satisfies the growth condition

$$
\int_{E(R)} f^{2} \exp (-2 a r)=o(R)
$$

as $R \rightarrow \infty$, then it must satisfy the decay estimate

$$
\int_{E(R+1) \backslash E(R)} f^{2} \leqslant C \exp (-2 a R)
$$

for some constant $C>0$ depending on $f$ and $a$.

Proof. - By setting $-V(x)=\mu$ we can rewrite the Poincaré inequality as

$$
\left(\lambda_{1}(E)-\mu\right) \int_{E} \phi^{2}(x) d x \leqslant \int_{E}|\nabla \phi|^{2}(x) d x-\mu \int_{E} \phi^{2}(x) d x .
$$

We now can apply Theorem 2.1 by setting $\rho=a^{2}$. The distance function with respect to the metric $\rho d s^{2}$ is then given by

$$
r_{\rho}(x)=\operatorname{ar}(x)
$$

where $r(x)$ is the background distance function to the smooth compact set $\Omega \subset M$. The corollary follows from Theorem 2.1.

COROLLARY 2.3. - Let $M$ be a complete Riemannian manifold satisfying property $\left(\mathcal{P}_{\rho}\right)$. Suppose $\left\{E_{1}, \ldots, E_{k}\right\}$ with $k \geqslant 2$ are the nonparabolic ends of $M$. Then for each $1 \leqslant i \leqslant k$ there exists a bounded harmonic function $f_{i}$ defined on $M$ satisfying the growth estimate

$$
\int_{B_{\rho}(R+1) \backslash B_{\rho}(R)}\left|\nabla f_{i}\right|^{2} \leqslant C \exp (-2 R) .
$$

Moreover, $0 \leqslant f_{i} \leqslant 1$ and has the property that

$$
\sup _{x \in E_{i}} f_{i}(x)=1
$$

and

$$
\inf _{x \in E_{j}} f_{i}(x)=0, \quad \text { for } j \neq i .
$$

Proof. - We will construct $f_{i}$ for the case $i=1$, and the construction for other values of $i$ is exactly the same. In this case, we will simply denote $f=f_{1}$. Following the theory of Li-Tam [10] (see also [11]), $f$ can be constructed by taking the limit, as $\bar{R} \rightarrow \infty$, of a converging subsequence of harmonic functions $f_{\bar{R}}$ satisfying

$$
\Delta f_{\bar{R}}=0 \quad \text { on } B(\bar{R})
$$

with boundary condition

$$
f_{\bar{R}}=1 \quad \text { on } \partial B(\bar{R}) \cap E_{1},
$$

$4^{\text {e }}$ SÉRIE - TOME $39-2006-\mathrm{N}^{\circ} 6$ 
and

$$
f_{\bar{R}}=0 \quad \text { on } \partial B(\bar{R}) \backslash E_{1} .
$$

In fact, we only need to verify the growth estimate for the Dirichlet integral for the limiting function. The other required properties of $f$ follow from the construction of $\mathrm{Li}-\mathrm{Tam}$. To check the growth estimate, we first show that on $\left(B_{\rho}(R+1) \backslash B_{\rho}(R)\right) \backslash E_{1}$, because of the boundary condition we can apply Theorem 2.1 to the function $f_{\bar{R}}$. By taking the limit, this implies that

$$
\int_{\left(B_{\rho}(R+1) \backslash B_{\rho}(R)\right) \backslash E_{1}} \rho f^{2} \leqslant C \exp (-2 R) .
$$

Similarly on $E_{1}$, we can apply Theorem 2.1 to the function $1-f_{\bar{R}}$, hence we obtain

$$
\int_{\left(E_{1} \cap B_{\rho}(R+1)\right) \backslash\left(E_{1} \cap B_{\rho}(R)\right)} \rho(1-f)^{2} \leqslant C \exp (-2 R) .
$$

Let us now consider the cut-off function

$$
\phi\left(r_{\rho}(x)\right)= \begin{cases}r_{\rho}(x)-R+1 & \text { for } R-1 \leqslant r_{\rho} \leqslant R, \\ 1 & \text { for } R \leqslant r_{\rho} \leqslant R+1, \\ R+2-r_{\rho} & \text { for } R+1 \leqslant r_{\rho} \leqslant R+2, \\ 0 & \text { otherwise. }\end{cases}
$$

Integrating by parts and Schwarz inequality yield

$$
\begin{aligned}
0 & =\int_{\left(B_{\rho}(R+2) \backslash B_{\rho}(R-1)\right) \backslash E_{1}} \phi^{2} f \Delta f \\
= & -\int_{\left(B_{\rho}(R+2) \backslash B_{\rho}(R-1)\right) \backslash E_{1}} \phi^{2}|\nabla f|^{2}-2 \\
& \leqslant-\frac{1}{2} \int_{\left(B_{\rho}(R+2) \backslash B_{\rho}(R-1)\right) \backslash E_{1}} \phi_{\left(B_{\rho}(R+2) \backslash B_{\rho}(R-1)\right) \backslash E_{1}} \phi f\langle\nabla \phi, \nabla f\rangle \\
\int_{\left(B_{\rho}(R+2) \backslash B_{\rho}(R-1)\right) \backslash E_{1}} & |\nabla \phi|^{2} f^{2} .
\end{aligned}
$$

Hence combining with the definition of $\phi$, we obtain the estimate

$$
\begin{aligned}
\int_{\left(B_{\rho}(R+1) \backslash B_{\rho}(R)\right) \backslash E_{1}}|\nabla f|^{2} & \leqslant \int_{\left(B_{\rho}(R+2) \backslash B_{\rho}(R-1)\right) \backslash E_{1}} \phi^{2}|\nabla f|^{2} \\
& \leqslant 4 \int_{\left(B_{\rho}(R+2) \backslash B_{\rho}(R-1)\right) \backslash E_{1}} \rho f^{2} .
\end{aligned}
$$

Applying the estimate (2.9) to the right-hand side, we conclude the desired estimate on the set $\left(B_{\rho}(R+1) \backslash B_{\rho}(R)\right) \backslash E_{1}$. The estimate on $\left(E_{1} \cap B_{\rho}(R+1)\right) \backslash\left(E_{1} \cap B_{\rho}(R)\right)$ follows by using the function $1-f$ and (2.10) instead.

We would like to point out that the hypothesis of Corollary 2.2, hence Theorem 2.1, is best possible. Indeed, if we consider the hyperbolic space form $\mathbb{H}^{n}$ of -1 constant sectional curvature, then the volume growth is given by

$$
V(R) \sim C \exp ((n-1) R)
$$


and

$$
\lambda_{1}\left(\mathbb{H}^{n}\right)=\frac{(n-1)^{2}}{4} .
$$

We consider Theorem 2.1 for the special case when $V(x)=0$ and $\rho=\frac{(n-1)^{2}}{4}$. In this case, the distance function $r_{\rho}$ with respect to the metric $\rho d s^{2}$ is simply given by

$$
r_{\rho}=\frac{(n-1)}{2} r
$$

where $r$ is the hyperbolic distance function. If $f$ is a nonconstant bounded harmonic function, then

$$
\int_{B(R)} \rho f^{2} \exp \left(-2 r_{\rho}\right)=O(R) .
$$

We claim that the conclusion of Theorem 2.1 is not valid, hence will imply that the hypothesis of Theorem 2.1 cannot be improved. Indeed, if the conclusion were true, then $f$ would be in $L^{2}\left(\mathbb{H}^{n}\right)$. However, Yau's theorem [22] implies that $f$ must be identically constant. On the other hand, it is known that $\mathbb{H}^{n}$ has an infinite dimensional space of bounded harmonic functions, which provides a contradiction.

Also note that in the case of $\mathbb{R}^{n}(n \geqslant 3)$, the distance function with respect to the $\rho$-metric is

$$
r_{\rho} \sim \frac{n-2}{2} \int_{1}^{r} t^{-1} d t=\log r^{\frac{n-2}{2}}
$$

as $r \rightarrow \infty$. If we consider a multiple of Green's function $f(x)=r^{2-n}$ on $\mathbb{R}^{n}$, then checking the hypothesis of Theorem 2.1 for $f(x)$ on $E=\mathbb{R}^{n} \backslash B(1)$, the integral

$$
\begin{aligned}
\int_{E} \rho f^{2} \exp \left(-2 r_{\rho}\right) & =\frac{(n-2)^{2}}{4} \int_{1}^{\infty} r^{-2} r^{-n+2} r^{4-2 n} r^{n-1} d r \\
& =\frac{(n-2)^{2}}{4} \int_{1}^{\infty} r^{-2 n+3} d r \\
& <\infty .
\end{aligned}
$$

Hence we can apply Theorem 2.1 to this choice of $f$. On the other hand, the integral

$$
\begin{aligned}
\int_{E_{\rho}(R+1) \backslash E_{\rho}(R)} \rho f^{2} & =\frac{(n-2)^{2}}{4} \int_{r_{\rho}=R+1}^{r_{\rho}=R} r^{-2} r^{4-2 n} r^{n-1} d r \\
& =\int_{e^{\frac{2 R}{n-2}}} r^{-n+1} d r \\
& =\frac{1}{n-2}(\exp (-2 R)-\exp (-2(R+1))) \\
& =\frac{1-e^{-2}}{n-2} \exp (-2 R) .
\end{aligned}
$$

$4^{\text {e }}$ SÉRIE - TOME $39-2006-\mathrm{N}^{\circ} 6$ 
This implies that the conclusion of Theorem 2.1 is also sharp in this case.

Finally, we point out that the preceding argument of Theorem 2.1 can be extended without much modification to deal with $p$-forms satisfying a suitable differential equation. We consider the operator

$$
\Delta+W(x)
$$

acting on the $p$-forms on $M$, where $\Delta$ is the Hodge Laplacian and $W$ an endomorphism on the bundle of $p$-forms on $M$.

THEOREM 2.4. - Let $M$ be a complete Riemannian manifold. Suppose $E$ is an end of $M$ such that there exists a nonnegative function $\rho(x)$ defined on $E$ with the property that

$$
\int_{E} \rho(x)|\eta|^{2}(x) d x \leqslant \int_{E}\left(|d \eta|^{2}(x)+|\delta \eta|^{2}(x)\right) d x+\int_{E}\langle W(\eta)(x), \eta(x)\rangle d x
$$

is valid for any compactly supported smooth p-form $\eta$ on E. Assume that the $\rho$-metric given by $d s_{\rho}^{2}=\rho d s_{M}^{2}$ is complete on $E$. Let $\omega$ be a smooth p-form defined on $E$ satisfying the differential inequality

$$
\langle(\Delta+W(x)) \omega, \omega\rangle(x) \leqslant 0
$$

for all $x \in E$. If $\omega$ satisfies the growth condition

$$
\int_{E_{\rho}(R)} \rho|\omega|^{2} \exp \left(-2 r_{\rho}\right)=o(R)
$$

as $R \rightarrow \infty$, then it must satisfy the decay estimate

$$
\int_{E_{\rho}(R+1) \backslash E_{\rho}(R)} \rho|\omega|^{2} \leqslant C \exp (-2 R)
$$

for some constant $C>0$ depending on $\omega$ and $\rho$.

\section{Geometric conditions for parabolicity and nonparabolicity}

In this section, we would like to discuss some geometric conditions for the parabolicity and nonparabolicity of an end $E$. In [11], we used the decay estimate similar to Section 2 to derive geometric conditions for parabolicity on a manifold with $\lambda_{1}(M)>0$. A similar argument will yield the following conditions for manifolds with property $\left(\mathcal{P}_{\rho}\right)$. The key issue is that when $\left(\mathcal{P}_{\rho}\right)$ is present, the geometric conditions involving $\rho$ for parabolicity and nonparabolicity has a substantial gap. This fact is important to the proof of our main theorems in the proceeding sections.

THEOREM 3.1. - Let $E$ be an end of a complete Riemannian manifold $M$ with property $\left(\mathcal{P}_{\rho}\right)$ for some weight function $\rho$. If $E$ is nonparabolic, then

$$
\int_{E_{\rho}(R+1) \backslash E_{\rho}(R)} \rho d V \geqslant C_{1} \exp (2 R)
$$


for some constant $C_{1}>0$ and for $R$ sufficiently large, where $E_{\rho}(R)=B_{\rho}(R) \cap E$. If $E$ is parabolic, then

$$
\int_{E} \rho d V<\infty
$$

and

$$
\int_{E \backslash E_{\rho}(R)} \rho d V \leqslant C_{2} \exp (-2 R),
$$

for some constant $C_{2}>0$ and for all $R$ sufficiently large.

Proof. - Following a similar argument as in the proof of Theorem 1.4 in [11] and applying the harmonic equation to the barrier function $f$ on a nonparabolic end, we obtain

$$
\begin{aligned}
C & =\int_{\partial E} \frac{\partial f}{\partial \nu} d A \\
& =\int_{\partial B_{\rho}(r) \cap E} \frac{\partial f}{\partial \nu} d A \\
& \leqslant\left(\int_{\partial B_{\rho}(r) \cap E}(\sqrt{\rho})^{-1}|\nabla f|^{2} d A\right)^{\frac{1}{2}}\left(\int_{\partial B_{\rho}(r) \cap E} \sqrt{\rho} d A\right)^{\frac{1}{2}} .
\end{aligned}
$$

On the other hand, the co-area formula asserts that

$$
\begin{aligned}
\int_{E_{\rho}(R+1) \backslash E_{\rho}(R)} h d V & =\int_{R}^{R+1} \int_{\partial B_{\rho}(r) \cap E} h\left|\nabla r_{\rho}\right|^{-1} d A d r_{\rho} \\
& =\int_{R}^{R+1} \int_{\partial B_{\rho}(r) \cap E} h(\sqrt{\rho})^{-1} d A d r_{\rho}
\end{aligned}
$$

for any measurable function $h$. Hence (3.1) together with Corollary 2.3 imply that

$$
\begin{aligned}
\int_{R}^{R+1}\left(\int_{\partial B_{\rho}(r) \cap E} \sqrt{\rho} d A\right)^{-1} d r_{\rho} & \leqslant C \int_{R}^{R+1} \int_{\partial B_{\rho}(r) \cap E}|\nabla f|^{2}(\sqrt{\rho})^{-1} d A d r_{\rho} \\
& =C \int_{E_{\rho}(R+1) \backslash E_{\rho}(R)}|\nabla f|^{2} d V \\
& \leqslant C \exp (-2 R) .
\end{aligned}
$$

Applying Schwarz inequality gives

$4^{\mathrm{e}}$ SÉRIE - TOME $39-2006-\mathrm{N}^{\circ} 6$ 


$$
\begin{aligned}
1 & \leqslant\left(\int_{R}^{R+1}\left(\int_{\partial B_{\rho}(r) \cap E} \sqrt{\rho} d A\right)^{-1} d r_{\rho}\right)\left(\int_{R}^{R+1} \int_{\partial B_{\rho}(r) \cap E} \sqrt{\rho} d A d r_{\rho}\right) \\
& \leqslant C \exp (-2 R) \int_{R}^{R+1} \int_{\partial B_{\rho}(r) \cap E} \sqrt{\rho} d A d r_{\rho} .
\end{aligned}
$$

Using the co-area formula again, we obtain the estimate

$$
C_{1} \exp (2 R) \leqslant \int_{E_{\rho}(R+1) \backslash E_{\rho}(R)} \rho d V
$$

as claimed.

If $E$ is parabolic, we apply the proof of Corollary 2.3 to the barrier function $f=1$ on $E$ and obtain

$$
\int_{E_{\rho}(R+1) \backslash E_{\rho}(R)} \rho d V \leqslant C \exp (-2 R)
$$

for all sufficiently large $R$. Summing over these estimates, we conclude that

$$
\begin{aligned}
\int_{E \backslash E_{\rho}(R)} \rho d V & =\sum_{i=0}^{\infty} \int_{E_{\rho}(R+i+1) \backslash E_{\rho}(R+i)} \rho d V \\
& \leqslant C \sum_{i=0}^{\infty} \exp (-2(R+i)) \\
& =C_{2} \exp (-2 R)
\end{aligned}
$$

for some constant $C_{2}>0$. This proves the second half of the theorem.

COROLlaRY 3.2. - Let $E$ be an end of a complete Riemannian manifold $M$ with property $\left(\mathcal{P}_{\rho}\right)$. If $E$ is nonparabolic then it must have at least quadratic volume growth. In particular, if the weight function $\rho$ satisfies

$$
\liminf _{x \rightarrow \infty} \rho(x)>0,
$$

then $E$ is nonparabolic if and only if $E$ has infinite volume.

Proof. - A theorem of Varopoulos [18] asserts that if $M$ is nonparabolic then

$$
\int_{1}^{\infty} A^{-1}(r) d r<\infty
$$

where $A(r)$ denotes the area of the boundary of the geodesic ball of radius $r$ centered at a fixed point. In fact, this criterion can be localized [9] at an end, namely, that an end $E$ is nonparabolic implies that

$$
\int_{1}^{\infty} A_{E}^{-1}(r) d r<\infty
$$


where $A_{E}(r)$ denotes the area of the set $\partial B(r) \cap E$. In particular, applying the Schwarz inequality, we conclude that

$$
\begin{aligned}
R & \leqslant\left(\int_{1}^{R} A_{E}^{-1}(r) d r\right)^{\frac{1}{2}}\left(\int_{1}^{R} A_{E}(r) d r\right)^{\frac{1}{2}} \\
& \leqslant C V_{E}^{\frac{1}{2}}(R),
\end{aligned}
$$

where $V_{E}(R)$ is the volume of $E \cap B(R)$. Hence $E$ must have infinite volume. If $E$ is parabolic, then Theorem 3.1 implies that

$$
\int_{E} \rho d V<\infty .
$$

The assumption that liminf $\rho>0$ implies that $E$ has finite volume.

COROLlaRY 3.3. - Let $E$ be an end of a complete Riemannian manifold $M$ with property $\left(\mathcal{P}_{\rho}\right)$. If $\rho(r)$ is the weight function that depends only on the distance $r$ to a fixed compact set, then $E$ is nonparabolic if and only if

$$
\int_{1}^{\infty} A_{E}^{-1}(r) d r<\infty .
$$

Proof. - As pointed out in the proof of Corollary 3.2, (3.3) is a necessary condition for nonparabolicity. We now assume that $M$ is parabolic and by Theorem 3.1

$$
\int_{E} \rho d V<\infty .
$$

However, applying the Schwarz inequality, we have

$$
\begin{aligned}
r_{\rho}(R) & =\int_{1}^{R} \sqrt{\rho}(r) d r \\
& \leqslant\left(\int_{1}^{R} \rho(r) A_{E}(r) d r\right)^{\frac{1}{2}}\left(\int_{1}^{R} A_{E}^{-1}(r) d r\right)^{\frac{1}{2}} .
\end{aligned}
$$

Letting $R \rightarrow \infty$ and using the completeness of the $\rho$-metric, we conclude that the left-hand side tends to infinity, hence

$$
\infty=\int_{1}^{\infty} A_{E}^{-1}(r) d r
$$

and the corollary is proved.

\section{Improved Bochner formula and metric rigidity}

In this section, we will recall an improved Bochner formula and consider the case when the inequality is realized as an equality. This formula computes the Laplacian of the gradient of

$4^{\text {e }}$ SÉRIE - TOME $39-2006-\mathrm{N}^{\circ} 6$ 
a harmonic function as in the standard Bochner formula, but with extra care taken on the Hessian term by using the harmonic equation one more time. This manipulation was first used effectively by Yau [21], and it is by now considered to be a standard trick.

LEMma 4.1. - Let $M^{n}$ be a complete Riemannian manifold of dimension $n \geqslant 2$. Assume that the Ricci curvature of $M$ satisfies the lower bound

$$
\operatorname{Ric}_{M}(x) \geqslant-(n-1) \tau(x)
$$

for all $x \in M$. Suppose $f$ is a nonconstant harmonic function defined on $M$. Then the function $|\nabla f|$ must satisfy the differential inequality

$$
\Delta|\nabla f| \geqslant-(n-1) \tau|\nabla f|+\frac{\left.|\nabla| \nabla f\right|^{2}}{(n-1)|\nabla f|}
$$

in the weak sense. Moreover, if equality holds, then $M$ is given by $M=\mathbb{R} \times N^{n-1}$ with the warped product metric

$$
d s_{M}^{2}=d t^{2}+\eta^{2}(t) d s_{N}^{2}
$$

for some positive function $\eta(t)$, and some manifold $N^{n-1}$. In this case, $\tau(t)$ is a function of $t$ alone satisfying

$$
\eta^{\prime \prime}(t) \eta^{-1}(t)=\tau(t)
$$

Proof. - If we denote

$$
g=|\nabla f|,
$$

then the Bochner formula (see Theorem 2.1 of [11]) and the lower bound of the Ricci curvature assert that

$$
\Delta g \geqslant-(n-1) \tau g+\frac{|\nabla g|^{2}}{(n-1) g} .
$$

Note that since $f$ is nonconstant, $g$ is not identically zero. Hence equality holds if and only if all the inequalities used in the proof of (4.1) are equalities. In particular, we conclude that there exists a function $\mu$, such that,

$$
f_{1 \alpha}=0
$$

and

$$
f_{\alpha \beta}=\mu \delta_{\alpha \beta}
$$

for all $\alpha, \beta=2, \ldots, n$, where $\left\{e_{1}, e_{2}, \ldots, e_{n}\right\}$ is an orthonormal frame satisfying $|\nabla f| e_{1}=\nabla f$ and $e_{\alpha} f=0$ for all $\alpha \neq 1$.

We can now argue to conclude that $M=\mathbb{R} \times N$ with the warped product metric

$$
d s^{2}=d t^{2}+\eta^{2}(t) d s_{N}^{2}
$$

for some manifold $N$ and for some positive function $\eta$. 
Indeed, since $\Delta f=0$, together with (4.2) and (4.3), the Hessian of $f$ must be of the form

$$
\left(f_{i j}\right)=\left(\begin{array}{ccccc}
-(n-1) \mu & 0 & 0 & \ldots & 0 \\
0 & \mu & 0 & \ldots & 0 \\
0 & 0 & \mu & \ldots & 0 \\
\vdots & \vdots & \vdots & \ddots & \\
0 & 0 & 0 & \ldots & \mu
\end{array}\right)
$$

The fact that $f_{1 \alpha}=0$ for all $\alpha \neq 1$ implies that $|\nabla f|$ is identically constant along the level set of $f$. In particular, the level sets of $|\nabla f|$ and $f$ coincide. We claim that $|\nabla f|$ does not vanish anywhere hence $f$ has no critical points and $g>0$. Assuming the contrary, if $|\nabla f|(x)=0$, by addition of a constant, we may assume $f(x)=0$. The regularity theory of harmonic function asserts that $f$ locally in a neighborhood of $x$ behaves like a homogeneous harmonic polynomial in $\mathbb{R}^{n}$ with the origin at $x$. This is impossible since the level set of $|\nabla f|$ and $f$ coincide. Hence $|\nabla f|>0$ and $M$ must be topologically the product $\mathbb{R} \times N$, where $N$ is given by the level set of $f$. Also,

$$
\begin{aligned}
\mu \delta_{\alpha \beta} & =f_{\alpha \beta} \\
& =h_{\alpha \beta} f_{1}
\end{aligned}
$$

with $\left(h_{\alpha \beta}\right)$ being the second fundamental form of the level set of $f$. Hence

$$
\begin{aligned}
f_{11} & =-(n-1) \mu \\
& =-H f_{1}
\end{aligned}
$$

where $H$ is the mean curvature of the level set of $f$. Note that since $e_{1}=\frac{\nabla f}{|\nabla f|}$, which is a globally defined vector field, we have

$$
f_{1}=|\nabla f| .
$$

The fact that $f$ and $|\nabla f|$ have the same level sets implies that there exists a function $\beta$, such that,

$$
f_{1}=\beta(f) .
$$

In particular,

$$
\begin{aligned}
f_{11} & =e_{1} e_{1} f-\nabla_{e_{1}} e_{1} f \\
& =\beta^{\prime}(f) f_{1}-\left\langle\nabla_{e_{1}} e_{1}, \nabla f\right\rangle \\
& =\beta^{\prime}(f) \beta(f)-|\nabla f|\left\langle\nabla_{e_{1}} e_{1}, e_{1}\right\rangle \\
& =\beta^{\prime}(f) \beta(f),
\end{aligned}
$$

hence $f_{11}$ has constant value along the level set of $f$. Combining with (4.5), we conclude that the level set of $f$ has constant mean curvature $H$. In particular, together with (4.4), this implies that the second fundamental form is given by

$$
h_{\alpha \beta}=\frac{H}{(n-1)|\nabla f|} \delta_{\alpha \beta},
$$

a constant multiple of the identity matrix along each level set of $f$. This implies the splitting of the metric given by the form

$$
d s_{M}^{2}=d t^{2}+\eta^{2}(t) d s_{N}^{2},
$$

$4^{\text {e }}$ SÉRIE - TOME $39-2006-\mathrm{N}^{\circ} 6$ 
with

$$
\begin{aligned}
(n-1) \frac{\eta^{\prime}}{\eta} & =H \\
& =-\frac{f_{11}}{f_{1}}
\end{aligned}
$$

Hence

$$
\eta^{n-1}=C_{1} f_{1}^{-1}
$$

and

$$
f=C_{1} \int_{0}^{t} \eta^{-(n-1)} d s+C_{2}
$$

for some constants $C_{1}$ and $C_{2}$.

In particular, $g=|\nabla f|$ implies that

$$
g=C_{1} \eta^{-(n-1)}(t)
$$

The equation

$$
\Delta g=-(n-1) \tau g+\frac{|\nabla g|^{2}}{(n-1) g}
$$

asserts that

$$
\frac{d^{2} g}{d t^{2}}+(n-1) \eta^{-1} \frac{d \eta}{d t} \frac{d g}{d t}=-(n-1) \tau g+\frac{|\nabla g|^{2}}{(n-1) g}
$$

hence

$$
\frac{d^{2} \eta}{d t^{2}}=\tau \eta
$$

This implies that $\tau(t)$ must be a function of $t$ alone.

We remark that the manifold $N$ is necessarily compact if $M$ has more than one end.

COROLlary 4.2. - Let $M^{n}$ be a complete Riemannian manifold of dimension $n \geqslant 3$. Suppose $M$ satisfies the weighted Poincaré inequality for some nonnegative function $\rho$, and suppose that the Ricci curvature of $M$ is bounded from below by

$$
\operatorname{Ric}_{M}(x) \geqslant-\frac{n-1}{n-2} \rho(x)
$$

for all $x \in M$. If the volume growth of $M$ satisfies

$$
V_{p}(R) \leqslant C R^{2(n-1)}
$$

then either

(1) $M$ has only one nonparabolic end; or

(2) $M$ is given by $M=\mathbb{R} \times N$ with the warped product metric

$$
d s_{M}^{2}=d t^{2}+\eta^{2}(t) d s_{N}^{2}
$$

ANNALES SCIENTIFIQUES DE L'ÉCOLE NORMALE SUPÉRIEURE 
for some positive function $\eta(t)$, and some compact manifold $N$. Moreover, $\rho(t)$ is a function of t alone satisfying

$$
(n-2) \eta^{\prime \prime} \eta^{-1}=\rho .
$$

Proof. - According to the theory of Li-Tam [10], if $M$ has more than one nonparabolic end, then one can construct a nonconstant bounded harmonic function $f$ with finite Dirichlet integral. On the other hand, applying Schwarz inequality and using the volume growth assumption, we have

$$
\begin{aligned}
\int_{B(2 R) \backslash B(R)}|\nabla f|^{\frac{2(n-2)}{n-1}} & \leqslant\left(\int_{B(2 R) \backslash B(R)}|\nabla f|^{2}\right)^{\frac{n-2}{n-1}}\left(V_{p^{\frac{1}{n-1}}}^{\frac{1}{2}}(2 R)\right) \\
& \leqslant C R^{2}\left(\int_{B(2 R) \backslash B(R)}|\nabla f|^{2}\right)^{\frac{n-2}{n-1}} .
\end{aligned}
$$

The fact that $f$ has finite Dirichlet integral implies that the right-hand side is $o\left(R^{2}\right)$.

Let us denote

$$
g=|\nabla f|^{\frac{n-2}{n-1}} .
$$

Then Lemma 4.1 and the lower bound of the Ricci curvature assert that

$$
\Delta g \geqslant-\rho g
$$

Let $\phi$ be a nonnegative compactly supported smooth function on $M$. Then

$$
\int_{M}|\nabla(\phi g)|^{2}=\int_{M}|\nabla \phi|^{2} g^{2}+2 \int_{M} \phi g\langle\nabla \phi, \nabla g\rangle+\int_{M} \phi^{2}|\nabla g|^{2} .
$$

The second term on the right-hand side can be written as

$$
\begin{aligned}
2 \int_{M} \phi g\langle\nabla \phi, \nabla g\rangle & =\frac{1}{2} \int_{M}\left\langle\nabla\left(\phi^{2}\right), \nabla\left(g^{2}\right)\right\rangle \\
& =-\int_{M} \phi^{2} g \Delta g-\int_{M} \phi^{2}|\nabla g|^{2} \\
& =\int_{M} \phi^{2} \rho g^{2}-\int_{M} \phi^{2}|\nabla g|^{2}-\int_{M} \phi^{2} g(\Delta g+\rho g) .
\end{aligned}
$$

Combining with (4.8) and property $\left(\mathcal{P}_{\rho}\right)$, this implies that

$$
\begin{aligned}
\int_{M} \phi^{2} \rho g^{2} & \leqslant \int_{M}|\nabla(\phi g)|^{2} \\
& =\int_{M} \phi^{2} \rho g^{2}+\int_{M}|\nabla \phi|^{2} g^{2}-\int_{M} \phi^{2} g(\Delta g+\rho g) .
\end{aligned}
$$

Hence, we have

$$
\int_{M} \phi^{2} g(\Delta g+\rho g) \leqslant \int_{M}|\nabla \phi|^{2} g^{2} .
$$

$4^{\text {e }}$ SÉRIE - TOME $39-2006-\mathrm{N}^{\circ} 6$ 
For $R_{i}>0$, let us choose $\phi$ to satisfy the properties that

$$
\phi(r(x))= \begin{cases}1 & \text { if } r \leqslant R_{i} \\ 0 & \text { if } r \geqslant 2 R_{i}\end{cases}
$$

and

$$
\left|\phi^{\prime}\right| \leqslant C R_{i}^{-1} \quad \text { if } R_{i} \leqslant r \leqslant 2 R_{i}
$$

for some constant $C>0$. Then the right-hand side of (4.9) can be estimated by

$$
\int_{M}|\nabla \phi|^{2} g^{2} \leqslant C R_{i}^{-2} \int_{B\left(2 R_{i}\right) \backslash B\left(R_{i}\right)} g^{2} .
$$

By the growth estimate of $g$ in (4.6), the right-hand side tends to 0 as $R_{i} \rightarrow \infty$. Hence we conclude that (4.7) must indeed be equality. The corollary now follows from the equality part of Lemma 4.1. Moreover, the manifold $N$ must be compact because $M$ is assumed to have two ends.

We remark that if the Ricci curvature bound in Corollary 4.2 instead satisfies

$$
\operatorname{Ric}_{M}(x) \geqslant-\rho(x),
$$

then the validity of the generalized Poincaré inequality with weight function $\rho(x)$ alone implies that every harmonic function of finite Dirichlet energy on $M$ must be constant. In particular, $M$ has only one nonparabolic end. Indeed, in this case, we have

$$
\Delta|\nabla u| \geqslant-\rho|\nabla u|
$$

for any harmonic function $u$. With $|\nabla u| \in L^{2}(M)$, the inequality must be an equality by a similar argument using the generalized Poincaré inequality. Going back to the Bochner formula, one sees then that $|\nabla u|$ is a constant. Since $M$ is nonparabolic, the volume of $M$ must be infinite. The fact that $u$ has finite Dirichlet energy forces $|\nabla u|=0$. So $u$ is constant.

This remark is applicable to the case when $M$ is a stable minimal hypersurface in a nonnegatively curved complete manifold, which recovers a result proved by Schoen and Yau [16]. Another case is when $M$ is a locally conformally flat manifold with scalar curvature $R \leqslant 0$. Then, according to [17], the following inequality holds if $M$ is simply connected.

$$
C(n)\left(\int_{M}|\phi|^{\frac{2 n}{n-2}}\right)^{\frac{n-2}{n}}+\int_{M} \frac{n-2}{4(n-1)}|R| \phi^{2} \leqslant \int_{M}|\nabla \phi|^{2}
$$

for all $\phi \in C_{c}^{\infty}(M)$, where $n=\operatorname{dim} M \geqslant 3$ and $C(n)>0$, is a constant depending on $n$. Note that, in particular, this implies that $M$ satisfies a generalized Poincaré inequality with weight function

$$
\rho=\frac{n-2}{4(n-1)}|R| .
$$

Also $M$ satisfies a Sobolev inequality, hence all the ends of $M$ must be nonparabolic by [3]. In conclusion, $M$ has only one end in this case provided that the Ricci curvature of $M$ satisfies

$$
\operatorname{Ric}_{M}(x) \geqslant-\rho(x)=\frac{n-2}{4(n-1)} R .
$$


However, this condition is meaningful only for $n \geqslant 6$. Compare this with Corollary 5.5 in the next section.

COROLlaRY 4.3. - Let $M^{n}$ be a complete Riemannian manifold of dimension $n \geqslant 3$. Suppose $M$ satisfies property $\left(\mathcal{P}_{\rho}\right)$ for some nonnegative function $\rho$, and suppose that the Ricci curvature of $M$ is bounded from below by

$$
\operatorname{Ric}_{M}(x) \geqslant-\frac{n-1}{n-2} \rho(x)
$$

for all $x \in M$. If $M$ admits a nonconstant harmonic function $f$ with growth estimate satisfying

$$
\int_{B_{\rho}\left(2 R_{i}\right) \backslash B_{\rho}\left(R_{i}\right)} \rho|\nabla f|^{\frac{2(n-2)}{n-1}}=o\left(R_{i}^{2}\right)
$$

for a sequence of $R_{i} \rightarrow \infty$, then $M$ is given by $M=\mathbb{R} \times N$ with the warped product metric

$$
d s_{M}^{2}=d t^{2}+\eta^{2}(t) d s_{N}^{2},
$$

for some positive function $\eta(t)$, and some manifold $N$. Moreover, $\rho(t)$ is a function of t alone satisfying

$$
(n-2) \eta^{\prime \prime} \eta^{-1}=\rho .
$$

Proof. - We use a similar argument as in Corollary 4.2, except that we choose the cut-off function $\phi$ to satisfy the properties that

$$
\phi\left(r_{\rho}(x)\right)= \begin{cases}1 & \text { if } r_{\rho} \leqslant R_{i} \\ 0 & \text { if } r_{\rho} \geqslant 2 R_{i}\end{cases}
$$

and

$$
\left|\phi^{\prime}\right| \leqslant C R_{i}^{-1} \quad \text { if } R_{i} \leqslant r_{\rho} \leqslant 2 R_{i}
$$

for some constant $C>0$. The right-hand side of (4.9) can now be estimated by

$$
\int_{M}|\nabla \phi|^{2} g^{2} \leqslant C R_{i}^{-2} \int_{B_{\rho}\left(2 R_{i}\right) \backslash B_{\rho}\left(R_{i}\right)} \rho g^{2} .
$$

The assumption on the growth rate of $g=|\nabla f|^{\frac{n-2}{n-1}}$ implies that this tends to 0 as $R_{i} \rightarrow \infty$. Hence the left-hand side of (4.9) must be identically 0 , and Lemma 4.1 implies the corollary.

\section{Rigidity and nonparabolic ends}

In this section, we consider a complete manifold, $M^{n}$, with property $\left(\mathcal{P}_{\rho}\right)$ for some nonzero weight function $\rho(x) \geqslant 0$ for all $x \in M$. Note that since the existence of the weight function $\rho$ is equivalent to $M$ being nonparabolic, $M$ must have at least one nonparabolic end. Assuming that $M$ has at least two nonparabolic ends, $E_{1}$ and $E_{2}$, then a construction of Li-Tam [10] asserts that one can construct a nonconstant bounded harmonic function with finite Dirichlet integral.

4e SÉRIE - TOME $39-2006-\mathrm{N}^{\circ} 6$ 
Indeed, the harmonic function $f$ can be constructed by taking a convergent subsequence of the harmonic functions $f_{R}$, as $R \rightarrow \infty$, satisfying

$$
\Delta f_{R}=0 \quad \text { on } B(R)
$$

with boundary conditions

$$
f_{R}=1 \quad \text { on } \partial B(R) \cap E_{1}
$$

and

$$
f_{R}=0 \quad \text { on } \partial B(R) \backslash E_{1} .
$$

Moreover, the maximum principle asserts that $0 \leqslant f_{R} \leqslant 1$ for all $R$, hence $0 \leqslant f \leqslant 1$. We will first prove a lemma concerning the function $f$.

LEMMA 5.1. - Let $M$ be a complete manifold with property $\left(\mathcal{P}_{\rho}\right)$. Let $f$ be a bounded harmonic function described above (also in Corollary 2.3) with the property that $0 \leqslant \inf f<$ $\sup f<\infty$. Let us denote the level set of $f$ at $t$ by

$$
\ell(t)=\{x \in M \mid f(x)=t\}
$$

for $\inf f<t<\sup f$ and we denote the set

$$
\mathcal{L}(a, b)=\{x \in M \mid a<f(x)<b\}
$$

for $\inf f<a<b<\sup f$. Then

$$
\int_{\mathcal{L}(a, b)}|\nabla f|^{2}=(b-a) \int_{\ell(b)}|\nabla f| .
$$

Moreover,

$$
\int_{\ell(b)}|\nabla f|=\int_{\ell(t)}|\nabla f|
$$

for all inf $f<t<\sup f$.

Proof. - Let us first observe that if $\phi$ is a nonnegative compactly supported function, then the co-area formula asserts that

$$
\int_{M} \phi|\nabla f|^{2} d V=\int_{\inf f}^{\sup f} \int_{\ell(t)} \phi|\nabla f| d A d t .
$$

Letting $\phi$ tend to the constant function 1 and using the fact that $f$ has finite Dirichlet integral, we conclude that

$$
\int_{\inf f}^{\sup f} \int_{\ell(t)}|\nabla f| d A d t<\infty
$$

ANNALES SCIENTIFIQUES DE L'ÉCOLE NORMALE SUPÉRIEURE 
In particular, we conclude that

$$
\int_{\ell(t)}|\nabla f| d A<\infty
$$

for almost all $\inf f<t<\sup f$.

Let us again denote $\phi$ as a nonnegative compactly supported function. Integrating by parts and using the fact that $f$ is harmonic, we obtain

$$
\begin{aligned}
\int_{\mathcal{L}(a, b)} \phi^{2}|\nabla f|^{2} & =\int_{\ell(b)} \phi^{2} f f_{\nu}-\int_{\ell(a)} \phi^{2} f f_{\nu}-2 \int_{\mathcal{L}(a, b)} \phi f\langle\nabla \phi, \nabla f\rangle \\
& =b \int_{\ell(b)} \phi^{2} f_{\nu}-a \int_{\ell(a)} \phi^{2} f_{\nu}-2 \int_{\mathcal{L}(a, b)} \phi f\langle\nabla \phi, \nabla f\rangle,
\end{aligned}
$$

where $\nu$ is the unit normal of $\ell(t)$ given by $\nu|\nabla f|=\nabla f$. Since $f_{\nu}=|\nabla f|$, we can write

$$
\int_{\mathcal{L}(a, b)} \phi^{2}|\nabla f|^{2}=b \int_{\ell(b)} \phi^{2}|\nabla f|-a \int_{\ell(a)} \phi^{2}|\nabla f|-2 \int_{\mathcal{L}(a, b)} \phi f\langle\nabla \phi, \nabla f\rangle .
$$

Let us choose

$$
\phi(x)= \begin{cases}1 & \text { on } B_{\rho}(R), \\ R+1-r_{\rho}(x) & \text { on } B_{\rho}(R+1) \backslash B_{\rho}(R), \\ 0 & \text { on } M \backslash B_{\rho}(R+1) .\end{cases}
$$

Then

$$
|\nabla \phi|= \begin{cases}\sqrt{\rho} & \text { on } B_{\rho}(R+1) \backslash B_{\rho}(R), \\ 0 & \text { otherwise. }\end{cases}
$$

The last term on the right-hand side of (5.1) can be estimated by

$$
\begin{aligned}
& \left|\int_{\mathcal{L}(a, b)} \phi f\langle\nabla \phi, \nabla f\rangle\right| \\
& \leqslant \int_{\mathcal{L}(a, b) \cap\left(B_{\rho}(R+1) \backslash B_{\rho}(R)\right)} \sqrt{\rho}|f||\nabla f| \\
& \leqslant\left(\int_{\mathcal{L}(a, b) \cap\left(B_{\rho}(R+1) \backslash B_{\rho}(R)\right)} \rho f^{2}\right)^{\frac{1}{2}}\left(\int_{\mathcal{L}(a, b) \cap\left(B_{\rho}(R+1) \backslash B_{\rho}(R)\right)}|\nabla f|^{2}\right)^{\frac{1}{2}} \\
& \quad \leqslant C \exp (-R)\left(\int_{\mathcal{L}(a, b) \cap\left(B_{\rho}(R+1) \backslash B_{\rho}(R)\right)} \rho f^{2}\right)^{\frac{1}{2}},
\end{aligned}
$$

where we have used the estimate provided by Corollary 2.3 .

Following the notation and the estimate of Corollary 2.3, let $E_{1}$ be the nonparabolic end on which $\sup f$ is achieved at infinity. Then on the set $\left(B_{\rho}(R+1) \backslash B_{\rho}(R)\right) \backslash E_{1}$, inequality (2.9) implies that

$$
\int_{\left(B_{\rho}(R+1) \backslash B_{\rho}(R)\right) \backslash E_{1}} \rho(f-\inf f)^{2} \leqslant C \exp (-2 R) .
$$

$4^{\mathrm{e}}$ SÉRIE - TOME $39-2006-\mathrm{N}^{\circ} 6$ 
In particular,

$$
\begin{aligned}
& \int_{\left(\mathcal{L}(a, b) \cap\left(B_{\rho}(R+1) \backslash B_{\rho}(R)\right)\right) \backslash E_{1}} \rho f^{2} \\
& \leqslant b^{2} \int_{\left(\mathcal{L}(a, b) \cap\left(B_{\rho}(R+1) \backslash B_{\rho}(R)\right)\right) \backslash E_{1}} \rho \\
& \leqslant b^{2}(a-\inf f)^{-2} \int_{\left(\mathcal{L}(a, b) \cap\left(B_{\rho}(R+1) \backslash B_{\rho}(R)\right)\right) \backslash E_{1}} \rho(f-\inf f)^{2} \\
& \leqslant b^{2}(a-\inf f)^{-2} C \exp (-2 R) .
\end{aligned}
$$

Similarly, on $E_{1}$, (2.10) implies that we have

$$
\begin{aligned}
& \int_{\mathcal{L}(a, b) \cap\left(B_{\rho}(R+1) \backslash B_{\rho}(R)\right) \cap E_{1}} \rho f^{2} \\
& \leqslant b^{2} \int_{\mathcal{L}(a, b) \cap\left(B_{\rho}(R+1) \backslash B_{\rho}(R)\right) \cap E_{1}} \rho \\
& \leqslant b^{2}(\sup f-b)^{-2} \int_{\left(B_{\rho}(R+1) \backslash B_{\rho}(R)\right) \cap E_{1}} \rho(\sup f-f)^{2} \\
& \leqslant b^{2}(\sup f-b)^{-2} C \exp (-2 R) .
\end{aligned}
$$

Together with (5.3) and (5.2), we conclude that

$$
\left|\int_{\mathcal{L}(a, b)} \phi f\langle\nabla \phi, \nabla f\rangle\right| \leqslant\left(b\left((a-\inf f)^{-1}+(\sup f-b)^{-1}\right)\right) C \exp (-2 R) .
$$

Letting $R \rightarrow \infty,(5.1)$ becomes

$$
\int_{\mathcal{L}(a, b)}|\nabla f|^{2}=b \int_{\ell(b)}|\nabla f|-a \int_{\ell(a)}|\nabla f| .
$$

We now observe that since (5.4) is independent of $\inf f$ and $\sup f$, if we apply (5.4) to the function $f+\varepsilon$, then we have

$$
\int_{\mathcal{L}(a, b)}|\nabla f|^{2}=(b+\varepsilon) \int_{\ell(b)}|\nabla f|-(a+\varepsilon) \int_{\ell(a)}|\nabla f| .
$$

Combining with (5.4), we conclude that

$$
\int_{\ell(a)}|\nabla f|=\int_{\ell(b)}|\nabla f|
$$

Since $a$ is arbitrary, this proves the lemma. 
We are now ready to prove the first main theorem. Let us first define

$$
S(R)=\sup _{B_{\rho}(R)} \sqrt{\rho}
$$

to be the supremum of $\sqrt{\rho}$ over the set $B_{\rho}(R)$.

THEOREM 5.2. - Let $M^{n}$ be a complete manifold with dimension $n \geqslant 3$. Assume that $M$ satisfies property $\left(\mathcal{P}_{\rho}\right)$ for some nonzero weight function $\rho \geqslant 0$. Suppose the Ricci curvature of $M$ satisfies the lower bound

$$
\operatorname{Ric}_{M}(x) \geqslant-\frac{n-1}{n-2} \rho(x)
$$

for all $x \in M$. If $\rho$ satisfies the growth estimate

$$
\liminf _{R \rightarrow \infty} \frac{S(R)}{F(R)}=0
$$

where

$$
F(R)= \begin{cases}\exp \left(\frac{n-3}{n-2} R\right) & \text { when } n \geqslant 4 \\ R & \text { when } n=3\end{cases}
$$

then either

(1) $M$ has only one nonparabolic end; or

(2) $M$ has two nonparabolic ends and is given by $M=\mathbb{R} \times N$ with the warped product metric

$$
d s_{M}^{2}=d t^{2}+\eta^{2}(t) d s_{N}^{2},
$$

for some positive function $\eta(t)$, and some compact manifold $N$. Moreover, $\rho(t)$ is a function of t alone satisfying

$$
(n-2) \eta^{\prime \prime} \eta^{-1}=\rho .
$$

Proof. - Let us assume that $M$ has at least two nonparabolic ends and that there exists a bounded harmonic function $f$ with finite Dirichlet integral constructed as above. We may assume that inf $f=0$ and $\sup f=1$. Note that the improved Bochner formula asserts that

$$
\Delta g \geqslant-\rho g
$$

where $g=|\nabla f|^{\frac{n-2}{n-1}}$, and according to Lemma 4.1, it suffices to show that

$$
\Delta g=-\rho g
$$

To see this, let us consider $\phi$ to be a nonnegative smooth function with compact support in $M$. Then

$$
\int_{M}|\nabla(\phi g)|^{2}=\int_{M}|\nabla \phi|^{2} g^{2}+2 \int_{M} \phi g\langle\nabla \phi, \nabla g\rangle+\int_{M} \phi^{2}|\nabla g|^{2} .
$$

The second term on the right-hand side can be written as

$$
2 \int_{M} \phi g\langle\nabla \phi, \nabla g\rangle=\frac{1}{2} \int_{M}\left\langle\nabla\left(\phi^{2}\right), \nabla\left(g^{2}\right)\right\rangle
$$

$4^{\mathrm{e}}$ SÉRIE - TOME $39-2006-\mathrm{N}^{\circ} 6$ 


$$
\begin{aligned}
& =-\int_{M} \phi^{2} g \Delta g-\int_{M} \phi^{2}|\nabla g|^{2} \\
& =\int_{M} \phi^{2} \rho g^{2}-\int_{M} \phi^{2}|\nabla g|^{2}-\int_{M} \phi^{2} g(\Delta g+\rho g) .
\end{aligned}
$$

Combining with (5.5) and property $\left(\mathcal{P}_{\rho}\right)$, this implies that

$$
\begin{aligned}
\int_{M} \phi^{2} \rho g^{2} & \leqslant \int_{M}|\nabla(\phi g)|^{2} \\
& =\int_{M} \phi^{2} \rho g^{2}+\int_{M}|\nabla \phi|^{2} g^{2}-\int_{M} \phi^{2} g(\Delta g+\rho g) .
\end{aligned}
$$

Hence, we have

$$
\int_{M} \phi^{2} g(\Delta g+\rho g) \leqslant \int_{M}|\nabla \phi|^{2} g^{2} .
$$

Let us choose $\phi=\psi \chi$ to be the product of two compactly supported functions. For $0<\delta<1$ and $0<\varepsilon<\frac{1}{2}$, let us choose $\chi$ to be

$$
\chi(x)= \begin{cases}0 & \text { on } \mathcal{L}(0, \delta \varepsilon) \cup \mathcal{L}(1-\delta \varepsilon, 1), \\ (-\log \delta)^{-1}(\log f-\log (\delta \varepsilon)) & \text { on } \mathcal{L}(\delta \varepsilon, \varepsilon) \cap\left(M \backslash E_{1}\right), \\ (-\log \delta)^{-1}(\log (1-f)-\log (\delta \varepsilon)) & \text { on } \mathcal{L}(1-\varepsilon, 1-\delta \varepsilon) \cap E_{1}, \\ 1 & \text { otherwise }\end{cases}
$$

For $R>0$, we choose

$$
\psi(x)= \begin{cases}1 & \text { on } B_{\rho}(R-1), \\ R-r_{\rho} & \text { on } B_{\rho}(R) \backslash B_{\rho}(R-1), \\ 0 & \text { on } M \backslash B_{\rho}(R) .\end{cases}
$$

Then applying to the right-hand side of (5.6), we obtain

$$
\int_{M}|\nabla \phi|^{2} g^{2} \leqslant 2 \int_{M}|\nabla \psi|^{2} \chi^{2}|\nabla f|^{\frac{2(n-2)}{n-1}}+2 \int_{M}|\nabla \chi|^{2} \psi^{2}|\nabla f|^{\frac{2(n-2)}{n-1}} .
$$

Let us now recall that, using the assumption on the Ricci curvature, the local gradient estimate of Cheng-Yau [4] (see [12]) for positive harmonic functions asserts that for all $R_{0}>0$,

$$
|\nabla f|(x) \leqslant\left((n-1) \sup _{B\left(x, R_{0}\right)} \sqrt{\rho(y)}+C R_{0}^{-1}\right) f(x),
$$

where $C$ is a constant depending only on $n$, and $B\left(x, R_{0}\right)$ is the ball of radius $R_{0}$ centered at $x$ with respect to the background metric $d s_{M}^{2}$. Let us now choose $R_{0}=\left(\sup _{B\left(x, R_{0}\right)} \sqrt{\rho}\right)^{-1}$. This choice of $R_{0}$ is possible as the function $r-\left(\sup _{B(x, r)} \sqrt{\rho}\right)^{-1}$ is negative when $r \rightarrow 0$ and it tends to $\infty$ as $r \rightarrow \infty$. Let us observe that if $y \in B\left(x, R_{0}\right)$, and if $\gamma$ is a $d s_{M}^{2}$ minimizing geodesic joining $x$ to $y$, then 


$$
\begin{aligned}
r_{\rho}(x, y) & =\int_{\gamma} \sqrt{\rho(\gamma(t))} d t \\
& \leqslant \sup _{B\left(x, R_{0}\right)} \sqrt{\rho(y)} R_{0} \\
& \leqslant 1 .
\end{aligned}
$$

This implies that $B\left(x, R_{0}\right) \subset B_{\rho}(x, 1)$. Hence (5.8) can be written as

$$
|\nabla f|(x) \leqslant C\left(\sup _{B_{\rho}(x, 1)} \sqrt{\rho}\right) f(x) .
$$

Similarly, applying the same estimate to $1-f$, we also have

$$
|\nabla f|(x) \leqslant C\left(\sup _{B_{\rho}(x, 1)} \sqrt{\rho}\right)(1-f(x)) .
$$

At the end $E_{1}$, the first term on the right-hand side of (5.7) can be estimated by

$$
\begin{aligned}
\int_{E_{1}}|\nabla \psi|^{2} \chi^{2}|\nabla f|^{\frac{2(n-2}{n-1}} & \leqslant \int_{\Omega} \rho|\nabla f|^{\frac{2(n-2)}{n-1}} \\
& \leqslant\left(\int_{\Omega}|\nabla f|^{2}\right)^{\frac{n-2}{n-1}}\left(\int_{\Omega} \rho^{n-1}\right)^{\frac{1}{n-1}},
\end{aligned}
$$

where

$$
\Omega=E_{1} \cap\left(B_{\rho}(R) \backslash B_{\rho}(R-1)\right) \cap(\mathcal{L}(\delta \varepsilon, 1-\delta \varepsilon)) .
$$

Applying Corollary 2.3, we conclude that

$$
\left(\int_{\Omega}|\nabla f|^{2}\right)^{\frac{n-2}{n-1}} \leqslant C \exp \left(-\frac{2(n-2) R}{n-1}\right) .
$$

On the other hand, using (2.10), we have

$$
\begin{aligned}
\int_{\Omega} \rho^{n-1} & \leqslant S^{2(n-2)}(R) \int_{\Omega} \rho \\
& \leqslant S^{2(n-2)}(R)(\delta \varepsilon)^{-2} \int_{\Omega} \rho(1-f)^{2} \\
& \leqslant C S^{2(n-2)}(R)(\delta \varepsilon)^{-2} \exp (-2 R) .
\end{aligned}
$$

Hence together with (5.11) and (5.12), we obtain

$$
\int_{E_{1}}|\nabla \psi|^{2} \chi^{2}|\nabla f|^{\frac{2(n-2)}{n-1}} \leqslant C S^{\frac{2(n-2)}{n-1}}(R)(\delta \varepsilon)^{-\frac{2}{n-1}} \exp (-2 R) .
$$

Using (5.10), the second term on the right-hand side of (5.7) at $E_{1}$ can be estimated by $4^{\text {e }}$ SÉRIE - TOME $39-2006-\mathrm{N}^{\circ} 6$ 


$$
\begin{aligned}
& \int_{E_{1}}|\nabla \chi|^{2} \psi^{2}|\nabla f|^{\frac{2(n-2)}{n-1}} \\
& \leqslant(\log \delta)^{-2} \int_{\mathcal{L}(1-\varepsilon, 1-\delta \varepsilon) \cap E_{1} \cap B_{\rho}(R)}|\nabla f|^{2+\frac{2(n-2)}{n-1}}(1-f)^{-2} \\
& \leqslant C S^{\frac{2(n-2)}{n-1}}(R+1)(\log \delta)^{-2} \int_{\mathcal{L}(1-\varepsilon, 1-\delta \varepsilon) \cap E_{1} \cap B_{\rho}(R)}|\nabla f|^{2}(1-f)^{\frac{2(n-2)}{n-1}-2} .
\end{aligned}
$$

Note that the co-area formula and Lemma 5.1 imply that

$$
\begin{gathered}
\int_{\mathcal{L}(1-\varepsilon, 1-\delta \varepsilon) \cap E_{1} \cap B_{\rho}(R)}|\nabla f|^{2}(1-f)^{\frac{2(n-2)}{n-1}-2} \\
\leqslant \int_{1-\varepsilon}^{1-\delta \varepsilon}(1-t)^{\frac{2(n-2)}{n-1}-2} \int_{\ell(t) \cap E_{1} \cap B_{\rho}(R)}|\nabla f| d A d t \\
\leqslant \int_{\ell(b)}|\nabla f| d A \int_{1-\varepsilon}^{1-\delta \varepsilon}(1-t)^{\frac{2(n-2)}{n-1}-2} d t
\end{gathered}
$$

for any level $b$. Since

$$
\int_{1-\varepsilon}^{1-\delta \varepsilon}(1-t)^{\frac{2(n-2)}{n-1}-2} d t= \begin{cases}\frac{n-1}{n-3}\left(\varepsilon^{\frac{n-3}{n-1}}-(\delta \varepsilon)^{\frac{n-3}{n-1}}\right) & \text { when } n \geqslant 4 \\ -\log \delta & \text { when } n=3\end{cases}
$$

together with (5.14), we conclude that

$$
\begin{aligned}
& \int_{E_{1}}|\nabla \chi|^{2} \psi^{2}|\nabla f|^{\frac{2(n-2)}{n-1}} \\
& \leqslant \begin{cases}C S^{\frac{2(n-2)}{n-1}}(R+1)(\log \delta)^{-2}\left(1-\delta^{\frac{n-3}{n-1}}\right) \varepsilon^{\frac{n-3}{n-1}} & \text { when } n \geqslant 4, \\
C S(R+1)(-\log \delta)^{-1} & \text { when } n=3 .\end{cases}
\end{aligned}
$$

When $n \geqslant 4$, we set $\delta=\frac{1}{2}$, (5.7), (5.13), and (5.15) together imply that

$$
\int_{E_{1}}|\nabla \phi|^{2} g^{2} \leqslant C S^{\frac{2(n-2)}{n-1}}(R+1)\left(\varepsilon^{-\frac{2}{n-1}} \exp (-2 R)+\varepsilon^{\frac{n-3}{n-1}}\right) .
$$

A similar argument using the function $f$ instead of $1-f$ and (5.9) instead of (5.10) will yield the estimate

$$
\int_{M \backslash E_{1}}|\nabla \phi|^{2} g^{2} \leqslant C S^{\frac{2(n-2)}{n-1}}(R+1)\left(\varepsilon^{-\frac{2}{n-1}} \exp (-2 R)+\varepsilon^{\frac{n-3}{n-1}}\right)
$$


on the other ends of $M$. In particular, we conclude that

$$
\int_{M}|\nabla \phi|^{2} g^{2} \leqslant C S^{\frac{2(n-2)}{n-1}}(R+1)\left(\varepsilon^{-\frac{2}{n-1}} \exp (-2 R)+\varepsilon^{\frac{n-3}{n-1}}\right)
$$

is valid for $R$ sufficiently large. Setting $\varepsilon=\exp (-2 R)$, this becomes

$$
\int_{M}|\nabla \phi|^{2} g^{2} \leqslant C S^{\frac{2(n-2)}{n-1}}(R+1) \exp \left(-\frac{2(n-3) R}{n-1}\right) .
$$

Hence the right-hand side tends to 0 by taking $R+1=R_{i}$ where $\lim _{R_{i} \rightarrow \infty} \frac{S\left(R_{i}\right)}{F\left(R_{i}\right)}=0$, and letting $i \rightarrow \infty$, and the theorem follows for $n \geqslant 4$.

Similarly, when $n=3$, we get

$$
\int_{M}|\nabla \phi|^{2} g^{2} \leqslant C S(R+1)\left(\delta^{-1} \varepsilon^{-1} \exp (-2 R)+(-\log \delta)^{-1}\right) .
$$

Set $\delta=\varepsilon=\exp (-R q(R))$ with

$$
q(R)=\sqrt{\frac{S(R+1)}{R}} .
$$

Note that the assumption that $\lim \inf \frac{S(R)}{R}=0$ implies that $q\left(R_{i}\right) \rightarrow 0$ for some sequence of $R_{i} \rightarrow \infty$. Hence

$$
\begin{aligned}
& S\left(R_{i}+1\right)\left(\delta^{-1} \varepsilon^{-1} \exp \left(-2 R_{i}\right)+(-\log \delta)^{-1}\right) \\
& \quad=S\left(R_{i}+1\right)\left(\exp \left(-2 R_{i}\left(1-q\left(R_{i}\right)\right)\right)+R_{i}^{-1} q^{-1}\left(R_{i}\right)\right) .
\end{aligned}
$$

For sufficiently large $i$, the term $q\left(R_{i}\right) \leqslant \frac{1}{2}$, and

$$
S\left(R_{i}+1\right) \exp \left(-2 R_{i}\left(1-q\left(R_{i}\right)\right)\right) \leqslant S\left(R_{i}+1\right) \exp \left(-R_{i}\right)
$$

must tend to 0 as $i \rightarrow \infty$. By definition of $q$, the other term

$$
S\left(R_{i}+1\right) R_{i}^{-1} q^{-1}\left(R_{i}\right)=q\left(R_{i}\right)
$$

also tends to 0 as remarked earlier. Hence the right-hand side of (5.16) tends to 0 and the theorem is proved.

Obviously, if $\rho$ is bounded, then the growth assumption on $\rho$ in the theorem is automatically satisfied. We claim this is also true when $\rho(r)$ is an nondecreasing function depending only on the distance to a compact set with respect to the background metric $d s_{M}^{2}$ and $n \geqslant 4$. Indeed, the $\rho$-distance to the compact set

$$
r_{\rho}(r)=\int_{0}^{r} \sqrt{\rho(t)} d t
$$

is also a function of $r$ alone and the function

$$
h(r)=\exp \left(-\frac{n-3}{n-2} r_{\rho}(r)\right) \rightarrow 0
$$

$4^{\mathrm{e}}$ SÉRIE - TOME $39-2006-\mathrm{N}^{\circ} 6$ 
as $r \rightarrow \infty$. So there exists $r_{i} \rightarrow \infty$ such that

$$
\liminf _{r \rightarrow \infty} h^{\prime}(r)=0
$$

which verifies the claim.

When $n=3$, if we assume $\rho(r)$ is nondecreasing and of subexponential growth in $r$, then the same argument applying to the function $\ln r_{\rho}(r)$ instead will show that the growth assumption of the theorem is satisfied.

COROLLARY 5.3. - Let $M^{n}$ be a complete manifold, of dimension $n \geqslant 4$, with property $\left(\mathcal{P}_{\rho}\right)$ for some nonzero weight function $\rho \geqslant 0$. Suppose the Ricci curvature of $M$ satisfies the lower bound

$$
\operatorname{Ric}_{M}(x) \geqslant-\frac{n-1}{n-2} \rho(x)
$$

for all $x \in M$. Assume that $\rho(x)=\rho(r(x))$ is a nondecreasing function of the distance $r$ to a fixed compact set with respect to the background metric $d s_{M}^{2}$. Then either

(1) $M$ has only one nonparabolic end; or

(2) $M$ is given by $M=\mathbb{R} \times N$ with the warped product metric

$$
d s_{M}^{2}=d t^{2}+\eta^{2}(t) d s_{N}^{2},
$$

for some positive function $\eta(t)$, and some compact manifold $N$. Moreover, $\rho(t)$ is a function of t alone satisfying

$$
(n-2) \eta^{\prime \prime} \eta^{-1}=\rho .
$$

In particular, if $\rho(r)$ is asymptotically a polynomial in $r$, then the conclusion of the theorem is valid.

COROLLARY 5.4. - Let $M^{3}$ be a complete manifold, of dimension 3 , with property $\left(\mathcal{P}_{\rho}\right)$ for some nonzero weight function $\rho \geqslant 0$. Suppose the Ricci curvature of $M$ satisfies the lower bound

$$
\operatorname{Ric}_{M}(x) \geqslant-2 \rho(x)
$$

for all $x \in M$. Assume that $\rho(r)$ is a nondecreasing function of the distance to a fixed compact set with respect to the background metric $d s_{M}^{2}$ and that $\rho(r)$ is of subexponential growth. Then either

(1) $M$ has only one nonparabolic end; or

(2) $M$ has two nonparabolic ends and is given by $M=\mathbb{R} \times N$ with the warped product metric

$$
d s_{M}^{2}=d t^{2}+\eta^{2}(t) d s_{N}^{2}
$$

for some positive function $\eta(t)$, and some compact manifold $N$. Moreover, $\rho(t)$ is a function of $t$ alone satisfying

$$
\eta^{\prime \prime} \eta^{-1}=\rho .
$$

In particular, if $\rho(r)$ is asymptotically a polynomial in $r$, then the conclusion of the theorem is valid.

When $M$ is an $n$-dimensional, locally conformally flat, simply connected manifold with nonpositive scalar curvature $R$, according to the remarks made after Corollary 4.2, all ends of $M$ 
are nonparabolic and $M$ satisfies a generalized Poincaré inequality with weight function $\rho$ given by (4.10). Applying Theorem 5.2, we have the following result.

COROLLARY 5.5. - Let $\left(M^{n}, d s_{M}^{2}\right)$ be a complete, simply connected, locally conformally flat manifold, of dimension $n \geqslant 4$, with nonpositive scalar curvature $R$. Suppose the Ricci curvature of $M$ satisfies the lower bound

$$
\operatorname{Ric}_{M}(x) \geqslant \frac{1}{4} R(x)
$$

for all $x \in M$. Assume that the conformal metric $|R| d s_{M}^{2}$ is also complete, and

$$
\liminf _{r \rightarrow \infty} \exp \left(-\frac{n-3}{n-2} r\right) \sup _{B_{|R|}(r)}|R|(x)=0
$$

where the supremum is taken over the ball of radius $r$, with respect to the metric $|R| d s_{M}^{2}$, centered at some fixed point $p \in M$. Then either

(1) $M$ has only one end; or

(2) $M$ is given by $M=\mathbb{R} \times N$ with the warped product metric

$$
d s_{M}^{2}=d t^{2}+\eta^{2}(t) d s_{N}^{2},
$$

for some positive function $\eta(t)$, and some compact manifold $N$. Moreover, $R$ is a function of t alone satisfying

$$
4(n-1) \eta^{\prime \prime} \eta^{-1}=-R
$$

\section{Warped product metrics}

Let $M^{n}=\mathbb{R} \times N^{n-1}$ be the product manifold endowed with the warped product metric

$$
d s_{M}^{2}=d t^{2}+\eta^{2}(t) d s_{N}^{2},
$$

where $d s_{N}^{2}$ is a given metric on the compact manifold $N$. Our purpose is to compute the curvature on $M$ and discuss the possibility of the existence of a weight function $\rho$ that is given by a multiple of the lower bound of the Ricci curvature.

Let $\left\{\bar{\omega}_{2}, \ldots, \bar{\omega}_{n}\right\}$ be an orthonormal coframe on $N$ with respect to $d s_{N}^{2}$. If we define $\omega_{1}=d t$ and $\omega_{\alpha}=\eta(t) \bar{\omega}_{\alpha}$ for $2 \leqslant \alpha \leqslant n$, then the set $\left\{\omega_{i}\right\}_{i=1}^{n}$ forms an orthonormal coframe of $M$ with respect to $d s_{M}^{2}$. The first structural equations assert that

$$
d \omega_{i}=\omega_{i j} \wedge \omega_{j},
$$

where $\omega_{i j}$ are the connection 1-forms with the property that

$$
\omega_{i j}=-\omega_{j i}
$$

On the other hand, direct exterior differentiation yields

$$
d \omega_{1}=0
$$

and

$4^{\text {e }}$ SÉRIE - TOME $39-2006-\mathrm{N}^{\circ} 6$ 


$$
\begin{aligned}
d \omega_{\alpha} & =\eta^{\prime} \omega_{1} \wedge \bar{\omega}_{\alpha}+\eta \bar{\omega}_{\alpha \beta} \wedge \bar{\omega}_{\beta} \\
& =-(\log \eta)^{\prime} \omega_{\alpha} \wedge \omega_{1}+\bar{\omega}_{\alpha \beta} \wedge \omega_{\beta},
\end{aligned}
$$

where $\bar{\omega}_{\alpha \beta}$ are the connection 1-forms on $N$ and $\eta^{\prime}$ is the derivative of $\eta$ with respect to $t$. Hence we conclude that the connection 1-forms are given by

$$
\begin{aligned}
\omega_{1 \alpha} & =-\omega_{\alpha 1} \\
& =(\log \eta)^{\prime} \omega_{\alpha}
\end{aligned}
$$

and

$$
\omega_{\alpha \beta}=\bar{\omega}_{\alpha \beta} .
$$

The second structural equations also assert that

$$
d \omega_{i j}-\omega_{i k} \wedge \omega_{k j}=\frac{1}{2} R_{i j k l} \omega_{l} \wedge \omega_{k}
$$

where $R_{i j k l}$ is the curvature tensor on $M$. Exterior differentiating (6.1) yields

$$
d \omega_{1 \alpha}=(\log \eta)^{\prime \prime} \omega_{1} \wedge \omega_{\alpha}+(\log \eta)^{\prime}\left(-(\log \eta)^{\prime} \omega_{\alpha} \wedge \omega_{1}+\bar{\omega}_{\alpha \beta} \wedge \omega_{\beta}\right) .
$$

Hence combining with (6.1) and (6.2), we have

$$
d \omega_{1 \alpha}-\omega_{1 \beta} \wedge \omega_{\beta \alpha}=\left((\log \eta)^{\prime \prime}+\left((\log \eta)^{\prime}\right)^{2}\right) \omega_{1} \wedge \omega_{\alpha} .
$$

Also, exterior differentiating (6.2) gives

$$
d \omega_{\alpha \beta}=d \bar{\omega}_{\alpha \beta},
$$

and

$$
\begin{aligned}
& d \omega_{\alpha \beta}-\omega_{\alpha 1} \wedge \omega_{1 \beta}-\omega_{\alpha \gamma} \wedge \omega_{\gamma \beta} \\
& \quad=d \bar{\omega}_{\alpha \beta}-\bar{\omega}_{\alpha \gamma} \wedge \bar{\omega}_{\gamma \beta}+\left((\log \eta)^{\prime}\right)^{2} \omega_{\alpha} \wedge \omega_{\beta} \\
& \quad=\frac{1}{2} \bar{R}_{\alpha \beta \gamma \tau} \bar{\omega}_{\tau} \wedge \bar{\omega}_{\gamma}+\left((\log \eta)^{\prime}\right)^{2} \omega_{\alpha} \wedge \omega_{\beta} \\
& \quad=\frac{1}{2} \bar{R}_{\alpha \beta \gamma \tau} \eta^{-2} \omega_{\tau} \wedge \omega_{\gamma}+\left((\log \eta)^{\prime}\right)^{2} \omega_{\alpha} \wedge \omega_{\beta},
\end{aligned}
$$

where $\bar{R}_{\alpha \beta \gamma \tau}$ is the curvature tensor on $N$. In particular, the sectional curvature of the two-plane section spanned by $e_{1}$ and $e_{\alpha}$ is given by

$$
K\left(e_{1}, e_{\alpha}\right)=-\left((\log \eta)^{\prime \prime}+\left((\log \eta)^{\prime}\right)^{2}\right) .
$$

Also the sectional curvature of the two plane section spanned by $e_{\alpha}$ and $e_{\beta}$ is given by

$$
K\left(e_{\alpha}, e_{\beta}\right)=\eta^{-2} \bar{K}\left(e_{\alpha}, e_{\beta}\right)-\left((\log \eta)^{\prime}\right)^{2},
$$

where $\bar{K}$ is the sectional curvature of $N$. Moreover the curvature tensor is given by

$$
R_{1 \alpha j k}= \begin{cases}(\log \eta)^{\prime \prime}+\left((\log \eta)^{\prime}\right)^{2} & \text { if } j=\alpha, k=1, \\ -(\log \eta)^{\prime \prime}-\left((\log \eta)^{\prime}\right)^{2} & \text { if } j=1, k=\alpha \\ 0 & \text { otherwise }\end{cases}
$$


and

$$
R_{\alpha \beta i j}= \begin{cases}\eta^{-2} \bar{R}_{\alpha \beta i j}+\left((\log \eta)^{\prime}\right)^{2}\left(\delta_{\alpha \tau} \delta_{\beta \gamma}-\delta_{\alpha \gamma} \delta_{\beta \tau}\right) & \text { if } i=\gamma, j=\tau, \\ 0 & \text { otherwise. }\end{cases}
$$

The Ricci curvature is then given by

$$
\begin{aligned}
\operatorname{Ric}_{1 j} & =\sum_{\alpha} R_{1 \alpha j \alpha} \\
& =-(n-1)\left((\log \eta)^{\prime \prime}+\left((\log \eta)^{\prime}\right)^{2}\right) \delta_{1 j} \\
& =-(n-1) \eta^{\prime \prime} \eta^{-1} \delta_{1 j},
\end{aligned}
$$

and

$$
\begin{aligned}
\operatorname{Ric}_{\alpha \beta} & =\sum_{\gamma \neq \alpha} R_{\alpha \gamma \beta \gamma}+R_{\alpha 1 \beta 1} \\
& =\eta^{-2} \overline{\operatorname{Ric}}_{\alpha \beta}-\left((\log \eta)^{\prime \prime}+(n-1)\left((\log \eta)^{\prime}\right)^{2}\right) \delta_{\alpha \beta},
\end{aligned}
$$

where $\overline{\mathrm{Ric}}_{\alpha \beta}$ is the Ricci tensor on $N$.

Let $\Delta$ be the Laplace operator defined on functions of $M$. When restricted to a function $f(t)$ depending only on the variable $t$, it has the expression

$$
\Delta f(t)=\frac{\partial^{2} f}{\partial t^{2}}+(n-1)(\log \eta)^{\prime} \frac{\partial f}{\partial t} .
$$

In particular, if we define

$$
f(t)=\int_{0}^{t} \eta^{-(n-1)} d s,
$$

then a direct computation yields that

$$
\Delta f=0
$$

Taking

$$
\begin{aligned}
g & =|\nabla f|^{\frac{n-2}{n-1}} \\
& =\eta^{-(n-2)},
\end{aligned}
$$

we have

$$
\begin{aligned}
\Delta g & =-(n-2) \eta^{\prime \prime} \eta^{-(n-1)} \\
& =-(n-2) \eta^{\prime \prime} \eta^{-1} g .
\end{aligned}
$$

According to Proposition 1.1, if

$$
\eta^{\prime \prime}>0
$$

then the function

$$
\rho=(n-2) \eta^{\prime \prime} \eta^{-1}
$$

is a weight function, and $M$ satisfies the corresponding weighted Poincaré inequality.

Obviously, (6.3) implies that

$$
\operatorname{Ric}_{1 j}=-\frac{n-1}{n-2} \rho \delta_{1 j} .
$$

$4^{\mathrm{e}}$ SÉRIE - TOME $39-2006-\mathrm{N}^{\circ} 6$ 
The condition that

$$
\operatorname{Ric}_{M} \geqslant-\frac{n-1}{n-2} \rho
$$

is then equivalent to

$$
\eta^{-2} \overline{\mathrm{Ric}}_{\alpha \beta}-\left((\log \eta)^{\prime \prime}+(n-1)\left((\log \eta)^{\prime}\right)^{2}\right) \delta_{\alpha \beta} \geqslant-(n-1) \eta^{-1} \eta^{\prime \prime} \delta_{\alpha \beta}
$$

because of (6.4). This can be rewritten as

$$
(n-2)(\log \eta)^{\prime \prime}+\eta^{-2} \operatorname{Ric}_{N} \geqslant 0 .
$$

Let us summarize the above computation in the following proposition.

PROPOSITION 6.1. - Let us consider the warped-product metric defined on $M^{n}=\mathbb{R} \times N^{n-1}$, given by the form

$$
d s_{M}^{2}=d t^{2}+\eta^{2}(t) d s_{N}^{2}
$$

for some positive function $\eta(t)$ depending on $t$ alone. If the warping function $\eta$ satisfies (6.7) and (6.8), then the warped product manifold $M$ will have the property that

$$
\operatorname{Ric}_{M} \geqslant-\frac{n-1}{n-2} \rho
$$

where

$$
\rho=(n-2) \eta^{\prime \prime} \eta^{-1}
$$

Moreover, $M$ will satisfy the weighted Poincaré inequality

$$
\int_{M} \rho \phi^{2} \leqslant \int_{M}|\nabla \phi|^{2}
$$

for any compactly supported function $\phi \in C_{c}^{\infty}(M)$. Also $f$ defined by (6.5) is a harmonic function with $g$ defined by (6.6) satisfying

$$
\Delta g=-\rho g,
$$

and

$$
\operatorname{Ric}(\nabla f, \nabla f)=-\frac{n-1}{n-2} \rho|\nabla f|^{2} .
$$

Example 6.2. - We will provide the following special examples for $\eta$. Let us consider

$$
\eta(t)=\cosh u(t)
$$

We will take $u(t)$ to be an odd function with the property that

$$
u(-t)=-u(t) .
$$

Let us first focus our computation on the positive half-line $[0, \infty)$. Direct differentiation gives

$$
\eta^{\prime}=u^{\prime} \sinh u
$$

ANNALES SCIENTIFIQUES DE L'ÉCOLE NORMALE SUPÉRIEURE 
and

$$
\eta^{\prime \prime}=u^{\prime \prime} \sinh u+\left(u^{\prime}\right)^{2} \cosh u .
$$

If we assume that $\operatorname{Ric}_{N} \geqslant-C$ for some constant $C \geqslant 0$, then the conditions (6.7) and (6.8) become

$$
u^{\prime \prime} \sinh u+\left(u^{\prime}\right)^{2} \cosh u>0
$$

and

$$
\begin{aligned}
(n-2)\left(\eta^{\prime \prime} \eta-\left(\eta^{\prime}\right)^{2}\right) & =(n-2)\left(u^{\prime \prime} \sinh u \cosh u+\left(u^{\prime}\right)^{2}\right) \\
& \geqslant C .
\end{aligned}
$$

Obviously, if we take any $u$ with the property that

$$
u^{\prime \prime} \geqslant 0
$$

and

$$
u^{\prime}>\sqrt{\frac{C}{n-2}}
$$

on $[0, \infty)$, then both of these conditions will be satisfied. The oddness of $u$ ensures that the conditions are valid also on $(-\infty, 0]$. Examples of such $u$ can be given by a smooth approximation of the function

$$
v(t)= \begin{cases}C_{1} t^{\alpha} & \text { for } 1 \leqslant t \\ C_{1} t & \text { for } 0 \leqslant t \leqslant 1\end{cases}
$$

for any value of $\alpha \geqslant 1$ and $C_{1}>\sqrt{\frac{C}{n-2}}$. In this case,

$$
\rho(t) \sim(n-2) \alpha^{2} C_{1}^{2} t^{2 \alpha-2}
$$

as $|t| \rightarrow \infty$, hence $M$ has property $\left(\mathcal{P}_{\rho}\right)$.

On the other hand, we will also show that for some cases of $\rho$, the warped product situation does not exist.

THEOREM 6.3. - Let $M^{n}=\mathbb{R} \times N^{n-1}$ be the warped-product manifold with metric given by

$$
d s_{M}^{2}=d t^{2}+\eta^{2}(t) d s_{N}^{2} .
$$

Suppose $M$ has property $\left(\mathcal{P}_{\rho}\right)$ with

$$
\rho=(n-2) \eta^{\prime \prime} \eta^{-1}
$$

and

$$
\operatorname{Ric}_{M} \geqslant-\frac{n-1}{n-2} \rho .
$$

Then on a nonparabolic end $E$,

$$
\liminf _{x \rightarrow \infty} \rho(x)>0,
$$

for $x \in E$.

$4^{\text {e }}$ SÉRIE - TOME $39-2006-\mathrm{N}^{\circ} 6$ 
Proof. - Combining (6.9) and (6.8), we have

$$
\frac{\rho}{n-2} \eta^{2}-\left(\eta^{\prime}\right)^{2} \geqslant-C
$$

if we assume that $(n-2)^{-1} \operatorname{Ric}_{N} \geqslant C$ for some constant $C$. Let $E$ be a nonparabolic end. By reparametrizing if necessary, we may assume that $E$ is given by $[0, \infty) \times N$. The geometric condition for nonparabolicity is then given by

$$
\int_{0}^{\infty} \eta^{-(n-1)} d t<\infty
$$

In particular, this implies that $\eta^{\prime}\left(t_{0}\right)>0$ for some $t_{0}>0$. Again by reparametrizing, we may assume that $\eta^{\prime}(0)>0$. Moreover, since $\eta$ is nonconstant and $\eta^{\prime}$ is monotonically nondecreasing as $t \rightarrow \infty$, we conclude that

$$
\eta^{\prime}(t)>0
$$

for $t>0$. Combining with (6.10), this implies that

$$
\eta^{\prime} \leqslant \sqrt{\frac{\rho}{n-2}} \eta+C_{0}
$$

where $C_{0}^{2}=\max \{C, 0\}$. Dividing through by $\eta$, and integrating over the interval $(0, t)$ yield

$$
\log \eta(t)-\log \eta(0) \leqslant \frac{r_{\rho}(t)}{\sqrt{n-2}}+C_{0} \int_{0}^{t} \eta^{-1}
$$

with

$$
r_{\rho}(t)=\int_{0}^{t} \sqrt{\rho}
$$

In particular, this implies

$$
\eta(t) \leqslant \eta(0) \exp \left(C_{0} \int_{0}^{t} \eta^{-1}\right) \exp \left(\frac{r_{\rho}(t)}{\sqrt{n-2}}\right) .
$$

Substituting back into (6.11), we obtain

$$
\eta^{\prime}(t) \leqslant \sqrt{\frac{\rho}{n-2}} \eta(0) \exp \left(C_{0} \int_{0}^{t} \eta^{-1}\right) \exp \left(\frac{r_{\rho}(t)}{\sqrt{n-2}}\right)+C_{0} .
$$

On the other hand, combining (6.9) and (6.11), we have

$$
\begin{aligned}
\eta^{\prime \prime} & \geqslant \frac{\rho}{n-2} \eta \\
& \geqslant \sqrt{\frac{\rho}{n-2}}\left(\eta^{\prime}-C_{0}\right) .
\end{aligned}
$$

ANNALES SCIENTIFIQUES DE L'ÉCOLE NORMALE SUPÉRIEURE 
Let us first assume that $\eta^{\prime}(t)>C_{0}$ as $t \rightarrow \infty$. Since $\eta^{\prime}$ is monotonically nondecreasing, there exists $t_{0}>0$ such that

$$
\eta^{\prime}(t)>C_{0} \quad \text { for } t \geqslant t_{0}
$$

and we can rewrite (6.13) as

$$
\left(\log \left(\eta^{\prime}-C_{0}\right)\right)^{\prime} \geqslant \sqrt{\frac{\rho}{n-2}}
$$

for $t \geqslant t_{0}$. Integrating from $t_{0}$ to $t$, we obtain

$$
\eta^{\prime}(t)-C_{0} \geqslant\left(\eta^{\prime}\left(t_{0}\right)-C_{0}\right) \exp \left(\frac{r_{\rho}(t)-r_{\rho}\left(t_{0}\right)}{\sqrt{n-2}}\right) .
$$

Comparing this with (6.12), we conclude that

$$
\left(\eta^{\prime}\left(t_{0}\right)-C_{0}\right) \exp \left(-\frac{r_{\rho}\left(t_{0}\right)}{\sqrt{n-2}}\right) \leqslant \sqrt{\frac{\rho}{n-2}} \eta(0) \exp \left(C_{0} \int_{0}^{t} \eta^{-1}\right) .
$$

Let us now assume the contrary that

$$
\liminf _{t \rightarrow \infty} \rho(t)=0
$$

If

$$
\liminf _{t \rightarrow \infty} \sqrt{\rho} \exp \left(C_{0} \int_{0}^{t} \eta^{-1}\right)=0,
$$

then this will provide a contradiction. In fact, since (6.14) implies that

$$
\begin{aligned}
C_{0} \int_{0}^{t} \eta^{-1} & <C_{0} \int_{0}^{t_{0}} \eta^{-1}+C_{0} \int_{t_{0}}^{t}\left(C_{0}\left(t-t_{0}\right)+\eta\left(t_{0}\right)\right)^{-1} \\
& \leqslant C_{1}+\log t
\end{aligned}
$$

for some constant $C_{1},(6.15)$ is fulfilled if

$$
\liminf _{t \rightarrow \infty} \rho(t) t^{2}=0
$$

On the other hand, if

$$
\liminf _{t \rightarrow \infty} \rho(t) t^{2}>0
$$

then there exists a constant $\beta>0$ such that

$$
\rho(t) \geqslant \beta t^{-2}
$$

for sufficiently large $t>0$. However, integrating $\eta^{\prime}(t)>C_{0}$ for $t \geqslant t_{0}$, we have

$$
\eta(t) \geqslant \eta\left(t_{0}\right)+C_{0}\left(t-t_{0}\right)
$$

$4^{\text {e }}$ SÉRIE - TOME $39-2006-\mathrm{N}^{\circ} 6$ 
Using this and (6.16) in (6.9), we conclude that

$$
\begin{aligned}
\eta^{\prime \prime} & =(n-2)^{-1} \rho \eta \\
& \geqslant C_{2} t^{-1}
\end{aligned}
$$

for some constant $C_{2}>0$ and for sufficiently large $t$. Hence

$$
\eta^{\prime}(t) \geqslant C_{2} \log t
$$

and

$$
\eta(t) \geqslant C_{2} t \log t
$$

for sufficiently large $t>0$. Using this estimate on (6.9) again, we have

$$
\eta^{\prime \prime} \geqslant C_{3} t^{-1} \log t
$$

Integrating again yields

$$
\eta^{\prime} \geqslant \frac{C_{3}}{2}(\log t)^{2}
$$

and

$$
\eta \geqslant \frac{C_{3}}{2} t(\log t)^{2}
$$

for sufficiently large $t$. Note that this estimate of $\eta$ implies that

$$
\int_{0}^{\infty} \eta^{-1}<\infty,
$$

hence the condition (6.15) is again satisfied as long as

$$
\liminf _{t \rightarrow \infty} \rho(t)=0
$$

Let us now consider the possibility that $\eta^{\prime}(t) \leqslant C_{0}$ for all $t$. In this case, this is equivalent to taking

$$
\begin{aligned}
\int_{0}^{\infty} \eta^{\prime \prime} & =\lim _{t \rightarrow \infty} \eta^{\prime}(t) \\
& =C_{3}
\end{aligned}
$$

for some constant $C_{3} \leqslant C_{0}$. Of course, this also implies that

$$
\eta(t) \sim C_{3} t
$$

as $t \rightarrow \infty$. Conversely, if we take $v(t)$ to be a nonnegative integrable function with

$$
\int_{0}^{\infty} v \leqslant C_{0},
$$

ANNALES SCIENTIFIQUES DE L'ÉCOLE NORMALE SUPÉRIEURE 
then one can take $\eta^{\prime \prime}=v$ and $\eta^{\prime}$ will satisfy

$$
\eta^{\prime} \leqslant C_{0}
$$

For this choice, one checks rather easily that both (6.7) and (6.8) are satisfied.

With the above discussion, we conclude that

$$
\liminf _{t \rightarrow \infty} \rho(t)>0
$$

unless $\eta^{\prime \prime}$ is integrable with

$$
\begin{aligned}
\int_{0}^{\infty} \eta^{\prime \prime} & =C_{3} \\
& \leqslant C_{0} .
\end{aligned}
$$

In this case, $\eta$ must be asymptotically a linear function with the property

$$
\eta(t) \sim C_{3} t
$$

as $t \rightarrow \infty$ for some constant $C_{3} \leqslant C_{0}$. The condition that $\eta^{\prime \prime}$ is integrable is equivalent to the condition that

$$
\int_{0}^{\infty} t \rho(t) d t<\infty
$$

which implies

$$
\liminf _{t \rightarrow \infty} t^{2} \rho(t)=0
$$

Also,

$$
\begin{aligned}
\int_{0}^{\infty} \sqrt{\rho} & \leqslant \sqrt{n-2}\left(\int_{0}^{\infty} \eta^{\prime \prime}\right)^{\frac{1}{2}}\left(\int_{0}^{\infty} \eta^{-2}\right)^{\frac{1}{2}}
\end{aligned}
$$

implies that the metric $d s_{\rho}^{2}=\rho d s^{2}$ is incomplete. This contradicts property $\left(\mathcal{P}_{\rho}\right)$.

Note that in the case when $\eta^{\prime \prime}$ is integrable, even though the $\rho$-metric is not complete, the volume growth of $M$ is polynomial of order $t^{n}$ and the finiteness theorem is still valid according to Corollary 4.2.

\section{Parabolic ends}

In this section, we consider the issue of number of parabolic ends. For a 3-manifold with property $\left(\mathcal{P}_{\rho}\right)$, one can also deal with the parabolic ends with the same assumption on the Ricci curvature as for the nonparabolic ends.

THEOREM 7.1. - Let $M^{3}$ be a complete manifold with property $\left(\mathcal{P}_{\rho}\right)$. Suppose the Ricci curvature of $M$ satisfies the lower bound

$$
\operatorname{Ric}_{M}(x) \geqslant-2 \rho(x)
$$

$4^{\mathrm{e}}$ SÉRIE - TOME $39-2006-\mathrm{N}^{\circ} 6$ 
for all $x \in M$. If $\rho$ satisfies the growth estimate

$$
\liminf _{R \rightarrow \infty}\left(R^{-1} S(R)\right)=0
$$

then either

(1) $M$ has only one end; or

(2) $M$ has two nonparabolic ends and is given by $M=\mathbb{R} \times N$ with the warped product metric

$$
d s_{M}^{2}=d t^{2}+\eta^{2}(t) d s_{N}^{2}
$$

for some positive function $\eta(t)$, and some compact manifold $N$. Moreover, $\rho(t)$ is a function of t alone satisfying

$$
\eta^{\prime \prime} \eta^{-1}=\rho .
$$

(3) $M$ has one nonparabolic end and one parabolic end and is given by $M=\mathbb{R} \times N$ with the warped product metric

$$
d s_{M}^{2}=d t^{2}+\eta^{2}(t) d s_{N}^{2},
$$

for some positive function $\eta(t)$, and some compact manifold $N$. Moreover, $\rho(t)$ is a function of t alone satisfying

$$
\eta^{\prime \prime} \eta^{-1}=\rho .
$$

Proof. - According to Theorem 5.2, $M$ either has only one nonparabolic end or it must be the warped product with two nonparabolic ends. Hence we may assume that $M$ has one nonparabolic end and also a parabolic end $E$. The theorem of Li-Tam [10], together with a result of Nakai [14], asserts that one can construct a positive harmonic function $f$ with the property that

$$
\lim _{x \rightarrow \infty} f(x)=\infty \quad \text { for } x \in E,
$$

and

$$
\liminf _{x \rightarrow \infty} f(x)=0 \quad \text { for } x \in M \backslash E .
$$

As in the case of Theorem 5.2, we consider the function $g=|\nabla f|^{\frac{1}{2}}$. In view of Lemma 4.1, we have

$$
\Delta g \geqslant-\rho g
$$

and the theorem follows by showing that

$$
\Delta g=-\rho g .
$$

Following a similar argument as in the proof of Theorem 5.2, the estimate on $M \backslash E$ for the term

$$
\int_{M \backslash E}|\nabla \phi|^{2} g^{2}
$$

can be shown to tend to 0 . We only need to deal with the term

$$
\int_{E}|\nabla \phi|^{2} g^{2} .
$$

ANNALES SCIENTIFIQUES DE L'ÉCOLE NORMALE SUPÉRIEURE 
Indeed, just like the case for the nonparabolic end, we just choose $\phi=\psi \chi$ where

$$
\chi(x)= \begin{cases}1 & \text { on } \mathcal{L}(0, T) \cap E, \\ (\log T)^{-1}(2 \log T-\log f) & \text { on } \mathcal{L}\left(T, T^{2}\right) \cap E, \\ 0 & \text { on } \mathcal{L}\left(T^{2}, \infty\right) \cap E,\end{cases}
$$

and

$$
\psi(x)= \begin{cases}1 & \text { on } B_{\rho}(R-1) \cap E, \\ R-r_{\rho}(x) & \text { on }\left(B_{\rho}(R) \backslash B_{\rho}(R-1)\right) \cap E, \\ 0 & \text { on } E \backslash B_{\rho}(R) .\end{cases}
$$

In this case,

$$
\int_{E}|\nabla \phi|^{2} g^{2} \leqslant 2 \int_{E}|\nabla \psi|^{2} \chi^{2}|\nabla f|+2 \int_{E}|\nabla \chi|^{2} \psi^{2}|\nabla f| .
$$

Using (5.9) and a similar argument, the first term can be estimated by

$$
\begin{aligned}
\int_{E}|\nabla \psi|^{2} \chi^{2}|\nabla f| & \leqslant \int_{\Omega} \rho|\nabla f| \\
& \leqslant S(R+1) \int_{\Omega} \rho f \\
& \leqslant S(R+1) T^{2} \int_{\left(B_{\rho}(R) \backslash B_{\rho}(R-1)\right) \cap E} \rho,
\end{aligned}
$$

where

$$
\Omega=\mathcal{L}\left(0, T^{2}\right) \cap\left(B_{\rho}(R) \backslash B_{\rho}(R-1)\right) \cap E
$$

and

$$
S(R+1)=\sup _{B_{\rho}(R+1)} \sqrt{\rho} .
$$

However, combining with the estimate from Theorem 3.1 we conclude that

$$
\int_{E}|\nabla \psi|^{2} \chi^{2}|\nabla f| \leqslant C S(R+1) T^{2} \exp (-2 R) .
$$

The second term can be estimated by

$$
\begin{aligned}
\int_{E}|\nabla \chi|^{2} \psi^{2}|\nabla f| & \leqslant(\log T)^{-2} \int_{\mathcal{L}\left(T, T^{2}\right) \cap B_{\rho}(R) \cap E}|\nabla f|^{3} f^{-2} \\
& \leqslant(\log T)^{-2} S(R+1) \int_{\mathcal{L}\left(T, T^{2}\right) \cap B_{\rho}(R) \cap E}|\nabla f|^{2} f^{-1} \\
& \leqslant(\log T)^{-2} S(R+1) \int_{T}^{T^{2}} t^{-1} \int_{\ell(t)}|\nabla f| d A d t \\
& =C(\log T)^{-1} S(R+1) .
\end{aligned}
$$

$4^{\text {e }}$ SÉRIE - TOME $39-2006-\mathrm{N}^{\circ} 6$ 
Combining with (7.1) and (7.2), we have

$$
\int_{E}|\nabla \phi|^{2} g^{2} \leqslant C S(R+1)\left((\log T)^{-1}+T^{2} \exp (-2 R)\right) .
$$

As in the proof of Theorem 5.2, the right-hand side tends to 0 for a sequence of $R_{i} \rightarrow \infty$ by choosing $T=\exp (R q(R))$ with $q(R)=\sqrt{R^{-1} S(R+1)}$, and using the assumption on $S(R)$.

We now turn to the case $n \geqslant 4$.

THEOREM 7.2. - Let $M^{n}$ be a complete manifold of dimension $n \geqslant 4$ with property $\left(\mathcal{P}_{\rho}\right)$. Suppose the Ricci curvature of $M$ satisfies the lower bound

$$
\operatorname{Ric}_{M}(x) \geqslant-\frac{4}{n-1} \rho(x)
$$

for all $x \in M$. If $\rho$ satisfies the property that

$$
\lim _{x \rightarrow \infty} \rho(x)=0,
$$

then $M$ has only one end.

Proof. - Since $\frac{4}{n-1}<\frac{n-1}{n-2}$ for $n \geqslant 4$, Theorem 5.2 asserts that if $M$ has more than one nonparabolic end then it must be given by the warped product and $\operatorname{Ric}_{11}=-\frac{n-1}{n-2} \rho$ which is impossible. Hence $M$ has exactly one nonparabolic end. Assuming that $M$ has another end $E$ that is parabolic, we construct a positive harmonic function $f$ similar to the case of Theorem 7.1. Again, we let $g=|\nabla f|^{\frac{1}{2}}$. Lemma 4.1 asserts that it satisfies

$$
\Delta g \geqslant-\frac{2}{n-1} \rho g-\frac{n-3}{n-1} g^{-1}|\nabla g|^{2} .
$$

Again, because of Lemma 4.1, we will show that this inequality is indeed an equality and conclude that $M=\mathbb{R} \times N$ with the warped product metric

$$
d s_{M}^{2}=d t^{2}+\eta^{2}(t) d s_{N}^{2} .
$$

Moreover, the Ricci curvature in the $\frac{\partial}{\partial t}$ direction satisfies

$$
\begin{aligned}
-\frac{4}{n-1} \rho & =\operatorname{Ric}_{11} \\
& =-(n-1) \eta^{\prime \prime} \eta^{-1} .
\end{aligned}
$$

Following the argument of Theorem 5.2, we consider a cut-off function $\phi$ and the integral (5.5). A similar argument shows that the second term on the right-hand side of (5.5) can be written as

$$
\begin{aligned}
2 \int_{M} \phi g\langle\nabla \phi, \nabla g\rangle & =\frac{1}{2} \int_{M}\left\langle\nabla\left(\phi^{2}\right), \nabla\left(g^{2}\right)\right\rangle=-\int_{M} \phi^{2} g \Delta g-\int_{M} \phi^{2}|\nabla g|^{2} \\
& =\frac{2}{n-1} \int_{M} \phi^{2} \rho g^{2}+\frac{n-3}{n-1} \int_{M} \phi^{2}|\nabla g|^{2}-\int_{M} \phi^{2}|\nabla g|^{2}-\int_{M} \phi^{2} g h
\end{aligned}
$$


where

$$
h=\Delta g+\frac{2}{n-1} \rho g+\frac{n-3}{n-1} g^{-1}|\nabla g|^{2} .
$$

Hence together with (5.5), we have

$$
\begin{aligned}
\int_{M}|\nabla(\phi g)|^{2}+\int_{M} \phi^{2} g h \\
\quad=\frac{2}{n-1} \int_{M} \phi^{2} \rho g^{2}+\frac{n-3}{n-1} \int_{M} \phi^{2}|\nabla g|^{2}+\int_{M}|\nabla \phi|^{2} g^{2} .
\end{aligned}
$$

Applying (5.5) again, we obtain

$$
\begin{aligned}
\int_{M}|\nabla(\phi g)|^{2}+\frac{n-1}{2} \int_{M} \phi^{2} g h \\
\quad=\int_{M} \phi^{2} \rho g^{2}-(n-3) \int_{M} \phi g\langle\nabla \phi, \nabla g\rangle+\int_{M}|\nabla \phi|^{2} g^{2} .
\end{aligned}
$$

Together with the weighted Poincaré inequality, this implies that

$$
\frac{n-1}{2} \int_{M} \phi^{2} g h \leqslant-(n-3) \int_{M} \phi g\langle\nabla \phi, \nabla g\rangle+\int_{M}|\nabla \phi|^{2} g^{2} .
$$

As in the proof of Theorem 5.2, we need to choose the cut-off function $\phi$ so that the right-hand side tends to 0 and the theorem follows.

Note that by a theorem of Nakai [14] (also see [15]), the positive harmonic function $f$ can be taken to be proper on the parabolic end $E$, i.e.,

$$
\liminf _{x \rightarrow \infty} f(x)=\infty
$$

for $x \in E$. To deal with the right-hand side of (7.4) on $E$, for any $\beta>1$, we define

$$
\phi= \begin{cases}1 & \text { on } \mathcal{L}(0, T) \cap E, \\ (\log \beta)^{-1}(\log \beta T-\log f) & \text { on } \mathcal{L}(T, \beta T) \cap E, \\ 0 & \text { on } \mathcal{L}(\beta T, \infty) \cap E .\end{cases}
$$

Integrating by parts, we have

$$
\begin{aligned}
-\int_{M} \phi g\langle\nabla \phi \nabla g\rangle & =-\frac{1}{4} \int_{\mathcal{L}(T, \beta T) \cap E}\left\langle\nabla \phi^{2}, \nabla g^{2}\right\rangle \\
& =\frac{1}{4} \int_{\mathcal{L}(T, \beta T) \cap E} \Delta\left(\phi^{2}\right) g^{2}+\frac{1}{2} \int_{\ell(T) \cap E} \phi_{\nu} g^{2},
\end{aligned}
$$

where $\nu$ is the unit normal to $\ell(t)$ given by $|\nabla f| \nu=\nabla f$. Using the definition of $\phi$, we obtain $4^{\mathrm{e}}$ SÉRIE - TOME $39-2006-\mathrm{N}^{\circ} 6$ 


$$
\begin{aligned}
\int_{\mathcal{L}(T, \beta T) \cap E} \Delta\left(\phi^{2}\right) g^{2}= & 2 \int_{\mathcal{L}(T, \beta T) \cap E}|\nabla \phi|^{2} g^{2}+2 \int_{\mathcal{L}(T, \beta T) \cap E} \phi \Delta \phi g^{2} \\
= & 2(\log \beta)^{-2} \int_{\mathcal{L}(T, \beta T) \cap E}|\nabla f|^{3} f^{-2} \\
& +2(\log \beta)^{-2} \int_{\mathcal{L}(T, \beta T) \cap E}(\log \beta T-\log f)|\nabla f|^{3} f^{-2} .
\end{aligned}
$$

Using the assumption on $\rho$ and the gradient estimate (5.9) for $f$, we conclude that there exists a constant $C>0$ such that

$$
|\nabla f|(x) \leqslant C \bar{S}(T, \beta T) f(x)
$$

for all $x \in \mathcal{L}(T, \beta T) \cap E$, where

$$
\bar{S}(T, \beta T)=\sup _{\mathcal{B}} \sqrt{\rho}
$$

with the supremum taken over the set

$$
\mathcal{B}=\left\{x \mid r_{\rho}(x, \mathcal{L}(T, \beta T) \cap E) \leqslant 1\right\} .
$$

Hence using the fact that $\int_{\ell(t)}|\nabla f|$ is a constant independent of $t$, together with (7.6), we obtain

$$
\begin{aligned}
& \int_{\mathcal{L}(T, \beta T) \cap E} \Delta\left(\phi^{2}\right) g^{2} \\
& \leqslant C \bar{S}(T, \beta T)(\log \beta)^{-2}\left(\int_{\mathcal{L}(T, \beta T) \cap E}|\nabla f|^{2} f^{-1}\right. \\
& \left.\quad+\int_{\mathcal{L}(T, \beta T) \cap E}(\log \beta T-\log f)|\nabla f|^{2} f^{-1}\right) \\
& =C \bar{S}(T, \beta T)(\log \beta)^{-2}\left(\int_{T}^{\beta T} t^{-1}+\int_{T}^{\beta T}(\log \beta T-\log t) t^{-1}\right) \\
& =C \bar{S}(T, \beta T)\left((\log \beta)^{-1}+1\right) .
\end{aligned}
$$

On the other hand, the last term on the right-hand side of (7.5) is given by

$$
\begin{aligned}
\frac{1}{2} \int_{\ell(T) \cap E} \phi_{\nu} g^{2} & \leqslant-\frac{1}{2}(\log \beta)^{-1} \int_{\ell(T) \cap E} f_{\nu} f^{-1}|\nabla f| \\
& \leqslant 0 .
\end{aligned}
$$

Combining with (7.5) and (7.7), we obtain

$$
-\int_{M} \phi g\langle\nabla \phi, \nabla g\rangle \leqslant C \bar{S}(T, \beta T)\left((\log \beta)^{-1}+1\right) .
$$

ANNALES SCIENTIFIQUES DE L'ÉCOLE NORMALE SUPÉRIEURE 
The second term on the right-hand side of (7.4) can be estimated similarly and we have

$$
\int_{M}|\nabla \phi|^{2} g^{2} \leqslant C \bar{S}(T, \beta T)(\log \beta)^{-1} .
$$

Setting $\beta=T$ and using the assumption that $\sqrt{\rho} \rightarrow 0$, we conclude that the right-hand side of (7.4) tends to 0 as $T \rightarrow \infty$.

Note that the properness of $f$ on $E$ implies that the sublevel set $\mathcal{L}(0, \varepsilon) \cap E=\emptyset$ for sufficiently small $\varepsilon$. By taking $M \backslash E$ as a nonparabolic end, we choose $\phi=\psi \chi$ as in Theorem 5.2. In particular, we set

$$
\chi(x)= \begin{cases}0 & \text { on } \mathcal{L}(0, \delta \varepsilon), \\ (-\log \delta)^{-1}(\log f-\log (\delta \varepsilon)) & \text { on } \mathcal{L}(\delta \varepsilon, \varepsilon), \\ 1 & \text { on } \mathcal{L}(\varepsilon, \infty) \cap(M \backslash E),\end{cases}
$$

and

$$
\psi(x)= \begin{cases}1 & \text { on } B_{\rho}(R-1), \\ R-r_{\rho} & \text { on } B_{\rho}(R) \backslash B_{\rho}(R-1), \\ 0 & \text { on } M \backslash B_{\rho}(R) .\end{cases}
$$

The second term on the right-hand side of (7.4) on $M \backslash E$ can be estimated exactly as in the case when $n=3$ in the proof of Theorem 5.2. To deal with the first term on the right-hand side of (7.4), we use

$$
-2 \int_{M} \phi g\langle\nabla \phi \nabla g\rangle=-\int_{M} \psi \chi^{2}\left\langle\nabla \psi, \nabla\left(g^{2}\right)\right\rangle-\int_{M} \psi^{2} \chi\left\langle\nabla \chi, \nabla\left(g^{2}\right)\right\rangle .
$$

The first term on the right-hand side of (7.8) can be estimated by

$$
\begin{aligned}
-\int_{M} \psi \chi^{2}\left\langle\nabla \psi, \nabla\left(g^{2}\right)\right\rangle & \leqslant \int_{M} \psi \chi^{2}|\nabla \psi|\left|\nabla\left(g^{2}\right)\right| \\
& \leqslant \int_{\Omega} \sqrt{\rho}\left|\nabla\left(g^{2}\right)\right| \\
& \leqslant\left(\int_{\Omega} \rho\right)^{\frac{1}{2}}\left(\int_{\Omega}\left|\nabla\left(g^{2}\right)\right|^{2}\right)^{\frac{1}{2}}
\end{aligned}
$$

where

$$
\Omega=\left(B_{\rho}(R) \backslash B_{\rho}(R-1)\right) \cap \mathcal{L}(\delta \varepsilon, \infty) \cap(M \backslash E) .
$$

Note that the estimate (2.9) asserts that

$$
(\delta \varepsilon)^{2} \int_{\Omega} \rho \leqslant C \exp (-2 R)
$$

Also, since the Bochner formula (Lemma 4.1) implies that

$$
\Delta\left(g^{2}\right) \geqslant-\frac{4}{n-1} \rho g^{2},
$$

$4^{e}$ SÉRIE - TOME $39-2006-\mathrm{N}^{\circ} 6$ 
if $\tau$ is a nonnegative compactly supported function, then

$$
\begin{aligned}
-\frac{4}{n-1} \int_{M} \tau^{2} \rho g^{4} & \leqslant \int_{M} \tau^{2}\left(g^{2}\right) \Delta\left(g^{2}\right) \\
& =-2 \int_{M} \tau g^{2}\left\langle\nabla \tau, \nabla\left(g^{2}\right)\right\rangle-\int_{M} \tau^{2}\left|\nabla\left(g^{2}\right)\right|^{2} \\
& \leqslant 2 \int_{M}|\nabla \tau|^{2} g^{4}-\frac{1}{2} \int_{M} \tau^{2}\left|\nabla\left(g^{2}\right)\right|^{2}
\end{aligned}
$$

hence

$$
\int_{M} \tau^{2}\left|\nabla\left(g^{2}\right)\right|^{2} \leqslant \frac{8}{n-1} \int_{M} \tau^{2} \rho g^{4}+4 \int_{M}|\nabla \tau|^{2} g^{4} .
$$

Let us set

$$
\tau= \begin{cases}0 & \text { on } B_{\rho}(R-2) \cup\left(M \backslash B_{\rho}(R+1)\right), \\ r_{\rho}-R+2 & \text { on } B_{\rho}(R-1) \backslash B_{\rho}(R-2), \\ 1 & \text { on } B_{\rho}(R) \backslash B_{\rho}(R-1), \\ R-r_{\rho}+1 & \text { on } B_{\rho}(R+1) \backslash B_{\rho}(R) .\end{cases}
$$

Then (7.11) implies

$$
\begin{aligned}
\int_{B_{\rho}(R) \backslash B_{\rho}(R-1)}\left|\nabla\left(g^{2}\right)\right|^{2} & \leqslant C \int_{B_{\rho}(R+1) \backslash B_{\rho}(R-2)} \rho g^{4} \\
& \leqslant C S^{2}(R+1) \int_{B_{\rho}(R+1) \backslash B_{\rho}(R-2)}|\nabla f|^{2},
\end{aligned}
$$

where $S(R+1)=\sup _{B_{\rho}(R+1)} \sqrt{\rho}$. Applying Corollary 2.3 to (7.12) and combining with (7.9) and (7.10), we conclude that

$$
-\int_{M} \psi \chi^{2}\left\langle\nabla \psi, \nabla\left(g^{2}\right)\right\rangle \leqslant C(\delta \varepsilon)^{-1} S(R+1) \exp (-2 R) .
$$

To estimate the second term on the right-hand side of (7.8), we integrate by parts and get

$$
\begin{aligned}
& -\int_{M} \psi^{2} \chi\left\langle\nabla \chi, \nabla\left(g^{2}\right)\right\rangle \\
& =-\int_{\mathcal{L}(\delta \varepsilon, \varepsilon)} \psi^{2} \chi\left\langle\nabla \chi, \nabla\left(g^{2}\right)\right\rangle \\
& =\int_{\mathcal{L}(\delta \varepsilon, \varepsilon)} \psi^{2} \chi \Delta \chi g^{2}+\int_{\mathcal{L}(\delta \varepsilon, \varepsilon)} \psi^{2} g^{2}|\nabla \chi|^{2} \\
& \quad+2 \int_{\mathcal{L}(\delta \varepsilon, \varepsilon)} \psi \chi\langle\nabla \psi, \nabla \chi\rangle g^{2}-\int_{\ell(\varepsilon)} \psi^{2} \chi \chi_{\nu} g^{2}+\int_{\ell(\delta \varepsilon)} \psi^{2} \chi \chi_{\nu} g^{2},
\end{aligned}
$$

where $|\nabla f| \nu=\nabla f$. Using the definition of $\chi$, the two boundary terms become 


$$
\begin{aligned}
-\int_{\ell(\varepsilon)} \psi^{2} \chi \chi_{\nu} g^{2}+\int_{\ell(\delta \varepsilon)} \psi^{2} \chi \chi_{\nu} g^{2} & =-(-\log \delta)^{-1} \int_{\ell(\varepsilon)} \psi^{2} f_{\nu} f^{-1} g^{2} \\
& \leqslant 0 .
\end{aligned}
$$

Hence (7.14) becomes

$$
\begin{aligned}
-\int_{M} \psi^{2} \chi\left\langle\nabla \chi, \nabla\left(g^{2}\right)\right\rangle \leqslant & \int_{\mathcal{L}(\delta \varepsilon, \varepsilon)} \psi^{2} \chi \Delta \chi g^{2}+2 \int_{\mathcal{L}(\delta \varepsilon, \varepsilon)} \psi^{2} g^{2}|\nabla \chi|^{2} \\
& +\int_{\mathcal{L}(\delta \varepsilon, \varepsilon)} \chi^{2}|\nabla \psi|^{2} g^{2} .
\end{aligned}
$$

We can write the term

$$
\begin{aligned}
\int_{\mathcal{L}(\delta \varepsilon, \varepsilon)} \psi^{2} \chi \Delta \chi g^{2} & =-(-\log \delta)^{-2} \int_{\mathcal{L}(\delta \varepsilon, \varepsilon) \cap B_{\rho}(R)} \psi^{2} g^{2}(\log f-\log \delta \varepsilon)|\nabla f|^{2} f^{-2} \\
& \leqslant 0 .
\end{aligned}
$$

As in the case of Theorem 5.2, the other term in (7.4), and the terms in (7.15) together have the estimate given by (5.16). Hence combining with (7.4), (7.13), (7.15) and (7.16), we conclude that

$$
\frac{n-1}{2} \int_{M} \phi^{2} g h \leqslant C S(R+1)\left((\delta \varepsilon)^{-1} \exp (-2 R)+(-\log \delta)^{-1}\right) .
$$

Since $\rho$ is bounded, by first letting $R \rightarrow \infty$ and then by setting $\delta=\varepsilon \rightarrow 0$, the right-hand side must tend to 0 . This proves that $h$ must be identically 0 and by Lemma 4.1, we conclude that $M$ is given by the warped product. At this point, we would like to point out that the argument at the nonparabolic end only requires that

$$
\liminf _{R \rightarrow \infty} S(R+1) \exp (-2 R)=0 .
$$

Note that using (7.3) and

$$
\operatorname{Ric}_{\alpha \alpha} \geqslant-\frac{4}{n-1} \rho,
$$

the same argument as in Theorem 6.3 asserts that

$$
\liminf _{x \rightarrow \infty} \rho(x)>0
$$

on the nonparabolic end. However, this contradicts the assumption on $\rho$ and the theorem follows.

\section{Nonexistence results for parabolic ends}

In this section, we will discuss some nonexistence and uniqueness results for the case when

$$
\operatorname{Ric}_{M}(x) \geqslant-\frac{4}{n-1} \rho(x)
$$

$4^{\text {e }}$ SÉRIE - TOME $39-2006-\mathrm{N}^{\circ} 6$ 
for a manifold with property $\left(\mathcal{P}_{\rho}\right)$. The first theorem gives some indication of why we cannot prove Theorem 7.2 without the stringent assumption on $\rho$. The second theorem gives a restriction on the warped product situation.

THEOREM 8.1. - Let $M^{n}$ be a complete manifold with property $\left(\mathcal{P}_{\rho}\right)$ of dimension $n \geqslant 4$. Suppose that the Ricci curvature of $M$ is bounded by

$$
\operatorname{Ric}_{M}(x) \geqslant-\frac{4}{n-1} \rho(x)
$$

for all $x \in M$. Assume that $\rho(x)=\rho(r(x))$ depends only on the distance function $r(x)$ to a smooth compact subset $\Omega \subset M$ and it satisfies the conditions

$$
\left(\rho^{-\frac{1}{4}}\right)^{\prime \prime}(r) \geqslant 0 \text { for all } r \geqslant r_{0}
$$

Then $\rho$ must be bounded from above at infinity.

Proof. - In terms of Fermi coordinates $(\theta, r)$ emanating from $\partial \Omega$, let us write the volume form of $M$ as

$$
d V=J(\theta, r) d \theta d r
$$

where $d \theta$ is the volume form of $\partial \Omega$. A standard variational argument (see [7]) asserts that if we set

$$
\psi(\theta, r)=J^{\frac{1}{n-1}}(\theta, r)
$$

then $\psi$ must satisfy the differential inequality

$$
\psi^{\prime \prime} \leqslant \frac{4}{(n-1)^{2}} \rho \psi
$$

where the derivatives are taken with respect to the $r$ variable. Let us consider the function

$$
g(r)=C \rho^{-\frac{1}{4}}(r) \exp \left(\frac{2}{n-1} r_{\rho}(r)\right) .
$$

Direct differentiation of $g$ and the fact that $r_{\rho}^{\prime}=\sqrt{\rho}$ imply

$$
\begin{aligned}
g^{\prime} & =-\frac{C}{4} \rho^{-\frac{5}{4}} \rho^{\prime} \exp \left(\frac{2}{n-1} r_{\rho}(r)\right)+\frac{2 C}{n-1} \sqrt{\rho} \rho^{-\frac{1}{4}} \exp \left(\frac{2}{n-1} r_{\rho}(r)\right) \\
& =\left(-\frac{1}{4} \rho^{\prime} \rho^{-1}+\frac{2}{n-1} \sqrt{\rho}\right) g
\end{aligned}
$$

and

$$
g^{\prime \prime}=\left(-\frac{1}{4} \rho^{\prime \prime} \rho^{-1}+\frac{5}{16}\left(\rho^{\prime}\right)^{2} \rho^{-2}+\frac{4}{(n-1)^{2}} \rho\right) g .
$$

The assumption on $\rho$ implies that

$$
g^{\prime \prime}(r) \geqslant \frac{4}{(n-1)^{2}} \rho g(r) \quad \text { for all } r \geqslant r_{0}
$$


Observe that since $\rho^{\frac{1}{2}}$ is positive, there exists $r_{1} \geqslant r_{0}$ such that $\left(\rho^{-\frac{1}{2}}\right)^{\prime}\left(r_{1}\right)>-\frac{4}{n-1}$. Renaming $r_{1}$ to be $r_{0}$ if necessary, we conclude that $g\left(r_{0}\right)>0$ and $g^{\prime}\left(r_{0}\right)>0$. Hence by taking $C>0$ sufficiently large, we may assume that

$$
g\left(r_{0}\right) \geqslant \psi\left(\theta, r_{0}\right)
$$

and

$$
g^{\prime}\left(r_{0}\right)>\psi^{\prime}\left(\theta, r_{0}\right)
$$

Moreover,

$$
(g(r)-\psi(\theta, r))^{\prime \prime} \geqslant \frac{4}{(n-1)^{2}} \rho(g(r)-\psi(\theta, r))
$$

for all $r \geqslant r_{0}$. This implies that

$$
g(r) \geqslant \psi(r)
$$

for all $r \geqslant r_{0}$ as otherwise $g-\psi$ will have a local positive maximum which will violate (8.1). In particular,

$$
J(\theta, r) \leqslant C \rho^{-\frac{n-1}{4}} \exp \left(2 r_{\rho}(r)\right) .
$$

Since $M$ is nonparabolic, it must have at least one nonparabolic end $E$. Theorem 3.1 implies that

$$
\int_{E_{\rho}(R+1) \backslash E_{\rho}(R)} \rho d V \geqslant C_{1} \exp (2 R) .
$$

Hence substituting the upper bound of the volume form (8.2) into this estimate, we obtain the inequality

$$
\int_{R}^{R+1} \rho^{-\frac{n-1}{4}+1} d r \geqslant C_{2}
$$

for some constant $C_{2}>0$. Using the identity

$$
\int_{R}^{R+1} \rho^{\frac{1}{2}} d r=1
$$

we conclude that

$$
\sup _{R \leqslant r\left(r_{\rho}\right) \leqslant R+1} \rho^{-\frac{n-3}{4}}(r) \geqslant C_{2}
$$

for all $R$. In particular, since $n \geqslant 4$, there exists a sequence $r_{i} \rightarrow \infty$ such that

$$
\rho^{-\frac{1}{4}}\left(r_{i}\right) \geqslant C_{3}^{\frac{1}{n-3}}
$$

We now claim that

$$
\rho^{-\frac{1}{4}}(r) \geqslant C_{3}^{\frac{1}{n-3}}
$$

$4^{\text {e }}$ SÉRIE - TOME $39-2006-\mathrm{N}^{\circ} 6$ 
for sufficiently large $r$. Indeed, if this is not the case, then we can find $r_{0}$ and $\bar{r}_{0}$ such that there exists $r_{i} \in\left[r_{0}, \bar{r}_{0}\right]$ with

$$
\rho^{-\frac{1}{4}}\left(r_{0}\right)<C_{3}^{\frac{1}{n-3}}
$$

and

$$
\rho^{-\frac{1}{4}}\left(\bar{r}_{0}\right)<C_{3}^{\frac{1}{n-3}} .
$$

However, the assumption that $\left(\rho^{-\frac{1}{4}}\right)^{\prime \prime} \geqslant 0$ implies that

$$
\rho^{-\frac{1}{4}}(r)<C_{3}^{\frac{1}{n-3}}
$$

on the interval $\left[r_{0}, \bar{r}_{0}\right]$ by the maximum principle. This violates inequality (8.3), hence the proposition follows.

THEOREM 8.2. - Let $M^{n}=\mathbb{R} \times N^{n-1}$ be the warped product manifold with metric given by

$$
d s_{M}^{2}=d t^{2}+\eta^{2}(t) d s_{N}^{2} .
$$

Assume that $M$ has property $\left(\mathcal{P}_{\rho}\right)$, and whose Ricci curvature is bounded by

$$
\operatorname{Ric}_{M}(x) \geqslant-\frac{4}{n-1} \rho(x)
$$

for all $x \in M$. If we assume that $n \geqslant 4$ and if we denote

$$
\inf _{\operatorname{Ric}_{N}}(x)=C_{N},
$$

where the infimum is taken over all points $x \in N$ and all tangent directions of $N$, then $C_{N} \geqslant 0$. Moreover if $C_{N}=0$, then $\rho$ must be identically constant given by $\rho=a^{2}$ for some $a>0$. In this case, $\eta(t)$ is given by either

$$
\eta(t)=\exp \left(a t+t_{0}\right)
$$

or

$$
\eta(t)=\exp \left(-a t+t_{0}\right)
$$

for some fixed $t_{0}$.

Proof. - According to the computation in Section 6, the function

$$
f(t)=\int_{0}^{t} \eta^{-(n-1)} d s
$$

is a harmonic function on $M$. Moreover, a direct computation yields that if $g=|\nabla f|^{\frac{1}{2}}$, then

$$
\begin{aligned}
\Delta g & =\left(\frac{\partial^{2}}{\partial t^{2}}+(n-1) \eta^{\prime} \eta^{-1} \frac{\partial}{\partial t}\right) \eta^{-\frac{n-1}{2}} \\
& =-\frac{n-1}{2} \eta^{\prime \prime} \eta^{-1} g-\frac{(n-1)(n-3)}{4}\left(\eta^{\prime}\right)^{2} \eta^{-2} g \\
& =\frac{1}{2} \operatorname{Ric}_{11} g-\frac{(n-1)(n-3)}{4}\left(\eta^{\prime}\right)^{2} \eta^{-2} g .
\end{aligned}
$$

ANNALES SCIENTIFIQUES DE L'ÉCOLE NORMALE SUPÉRIEURE 
On the other hand, using (6.3), (6.4) can be written as

$$
\begin{aligned}
\operatorname{Ric}_{\alpha \alpha} & =-\eta^{\prime \prime} \eta^{-1}-(n-2)\left(\eta^{\prime}\right)^{2} \eta^{-2}+\eta^{-2} \overline{\operatorname{Ric}}_{\alpha \alpha} \\
& =\frac{1}{n-1} \operatorname{Ric}_{11}-(n-2)\left(\eta^{\prime}\right)^{2} \eta^{-2}+\eta^{-2} \overline{\operatorname{Ric}}_{\alpha \alpha}
\end{aligned}
$$

for $2 \leqslant \alpha \leqslant n$. Substituting this into (8.4), we have

$$
\Delta g=\frac{(n-1)}{4(n-2)} \operatorname{Ric}_{11} g+\frac{(n-1)(n-3)}{4(n-2)} \operatorname{Ric}_{\alpha \alpha} g-\frac{(n-1)(n-3)}{4(n-2)} \overline{\operatorname{Ric}}_{\alpha \alpha} \eta^{-2} g .
$$

Using the lower bound of the Ricci curvature, we can write this as

$$
\Delta g \geqslant-\rho g-\frac{(n-1)(n-3)}{4(n-2)} \overline{\operatorname{Ric}}_{\alpha \alpha} \eta^{-2} g
$$

Observe that when $n=3$, the term $\operatorname{Ric}_{\alpha \alpha}$ does not appear and the lower bound of the Ricci curvature has only been applied to the Ric $\mathrm{Ri}_{11}$ term.

After taking the infimum over all points $\bar{x} \in N$ and $\alpha$, then (8.5) becomes

$$
\Delta g \geqslant-\rho g-\frac{(n-1)(n-3)}{4(n-2)} C_{N} \eta^{-2} g
$$

Let us assume that $C_{N} \leqslant 0$. Then (8.6) implies that

$$
\Delta g \geqslant-\rho g
$$

Since $g=\eta^{-\frac{n-1}{2}}$, the integral

$$
\begin{aligned}
\int_{-t}^{t} g^{2} d V & =\int_{-t}^{t} \eta^{-(n-1)} \eta^{n-1} d t \\
& =2 t \\
& =o\left(t^{2}\right) .
\end{aligned}
$$

Hence, we can apply the argument similar to the proof of Corollary 4.3 to conclude that (8.7) is indeed an equality. In particular, since $n \geqslant 4$, we conclude that $C_{N}=0$,

$$
\begin{aligned}
-\frac{4}{n-1} \rho & =\operatorname{Ric}_{11} \\
& =-(n-1) \eta^{\prime \prime} \eta^{-1},
\end{aligned}
$$

and

$$
\begin{aligned}
-\frac{4}{n-1} \rho & =\operatorname{Ric}_{\alpha \alpha} \\
& =-\eta^{\prime \prime} \eta^{-1}-(n-2)\left(\eta^{\prime}\right)^{2} \eta^{-2} .
\end{aligned}
$$

In particular, this implies that

$$
\eta^{\prime \prime} \eta^{-1}=\left(\eta^{\prime}\right)^{2} \eta^{-2}
$$

$4^{\text {e }}$ SÉRIE - TOME $39-2006-\mathrm{N}^{\circ} 6$ 
and hence $\log \eta$ is a linear function. Plugging this back into (8.9), we conclude that $\rho$ is a constant. This proves the theorem.

\section{Acknowledgements}

The authors would like to thank S.T. Yau for his interest in this work and his encouragement for us to continue our effort in considering the general case when $\rho$ is not only a function of the distance.

\section{REFERENCES}

[1] Agmon S., Lectures on Exponential Decay of Solutions of Second-Order Elliptic Equations: Bounds on Eigenfunctions of $N$-Body Schrödinger Operators, Mathematical Notes, vol. 29, Princeton University Press, Princeton, NJ, 1982.

[2] Cai M., Galloway G.J., Boundaries of zero scalar curvature in the ADS/CFT correspondence, $A d v$. Theor. Math. Phys. 3 (1999) 1769-1783.

[3] CAO H., SHEN Y., ZHU S., The structure of stable minimal hypersurfaces in $\mathbb{R}^{n+1}$, Math. Res. Lett. 4 (1997) 637-644.

[4] Cheng S.Y., YAU S.T., Differential equations on Riemannian manifolds and their geometric applications, Comm. Pure Appl. Math. 28 (1975) 333-354.

[5] Fefferman C., Phong D.H., The uncertainty principle and sharp Gårding inequalities, Comm. Pure Appl. Math. 34 (1981) 285-331.

[6] Fefferman C., Phong D.H., Lower bounds for Schrödinger equations, in: Conference on Partial Differential Equations (Saint Jean de Monts, 1982), Conf. No. 7, Soc. Math. France, Paris, 1982, 7 pp.

[7] Li P., Lecture Notes on Geometric Analysis, Lecture Notes Series, vol. 6, Research Institute of Mathematics and Global Analysis Research Center, Seoul National University, Seoul, 1993.

[8] Li P., Curvature and function theory on Riemannian manifolds, in: Surveys in Differential Geometry: Papers Dedicated to Atiyah, Bott, Hirzebruch, and Singer, vol. VII, International Press, Cambridge, 2000, pp. 375-432.

[9] Li P., TAM L.F., Complete surfaces with finite total curvature, J. Diff. Geom. 33 (1991) 139-168.

[10] Li P., TAM L.F., Harmonic functions and the structure of complete manifolds, J. Diff. Geom. 35 (1992) 359-383.

[11] Li P., WANG J., Complete manifolds with positive spectrum, J. Diff. Geom. 58 (2001) 501-534.

[12] Li P., WANG J., Complete manifolds with positive spectrum, II , J. Diff. Geom. 62 (2002) 143-162.

[13] Li P., WANG J., Comparison theorem for Kähler manifolds and positivity of spectrum, J. Diff. Geom. 69 (2005) 43-74.

[14] NaKai M., On Evans potential, Proc. Japan Acad. 38 (1962) 624-629.

[15] NAPIER T., RAMACHANDRAN M., Structure theorems for complete Kähler manifolds and applications to Lefschetz type theorems, Geom. Funct. Anal. 5 (1995) 809-851.

[16] SCHOEN R., YAU S.T., Harmonic maps and the topology of stable hypersurfaces and manifolds with non-negative Ricci curvature, Comm. Math. Helv. 39 (1981) 333-341.

[17] Schoen R., YAu S.T., Conformally flat manifolds, Kleinian groups and scalar curvature, Invent. Math. 92 (1988) 47-71.

[18] VAROpoulos N., Potential theory and diffusion on Riemannian manifolds, in: Conference on Harmonic Analysis in Honor of Antoni Zygmund, vols. I, II, in: Wadsworth Math. Ser., Wadsworth, Belmont, CA, 1983, pp. 821-837.

[19] WANG X., On conformally compact Einstein manifolds, Math. Res. Lett. 8 (2001) 671-688.

[20] WitTen E., YAU S.T., Connectness of the boundary in the ADS.CFT correspondence, Adv. Theor. Math. Phys. 3 (1999) 1635-1655. 
[21] Yau S.T., Harmonic functions on complete Riemannian manifolds, Comm. Pure Appl. Math. 28 (1975) 201-228.

[22] YAU S.T., Some function-theoretic properties of complete Riemannian manifold and their applications to geometry, Indiana Univ. Math. J. 25 (1976) 659-670.

(Manuscrit reçu le 9 novembre 2005; accepté, après révision, le 27 novembre 2006.)

Peter LI

Department of Mathematics, University of California, Irvine, CA 92697-3875, USA

E-mail: pli@math.uci.edu

Jiaping WANG

School of Mathematics,

University of Minnesota,

Minneapolis, MN 55455, USA

E-mail: jiaping@math.umn.edu 